\title{
The Search for Muon Neutrinos from Northern Hemisphere Gamma-Ray Bursts with AMANDA
}

A. Achterberg ${ }^{1}$, M. Ackermann ${ }^{2}$, J. Adams ${ }^{3}$, J. Ahrens ${ }^{4}$, K. Andeen ${ }^{5}$, J. Auffenberg ${ }^{14}$, J. N. Bahcall ${ }^{7,34}$, X. Bai ${ }^{8}$, B. Baret ${ }^{9}$, S. W. Barwick ${ }^{11}$, R. Bay $^{12}$, K. Beattie ${ }^{13}$, T. Becka ${ }^{4}$, J. K. Becker ${ }^{10}$, K.-H. Becker ${ }^{14}$, P. Berghaus ${ }^{15}$, D. Berley ${ }^{16}$, E. Bernardini ${ }^{2}$, D. Bertrand ${ }^{15}$, D. Z. Besson ${ }^{17}$, E. Blaufuss ${ }^{16}$, D. J. Boersma ${ }^{5}$, C. Bohm ${ }^{18}$, J. Bolmont ${ }^{2}$, S. Böser ${ }^{2}$, O. Botner ${ }^{19}$, A. Bouchta ${ }^{19}$, J. Braun ${ }^{5}$, C. Burgess ${ }^{18}$, T. Burgess ${ }^{18}$, T. Castermans ${ }^{20}$, D. Chirkin ${ }^{13}$, B. Christy ${ }^{16}$, J. Clem ${ }^{8}$, D. F. Cowen ${ }^{6,21}$, M. V. D'Agostino ${ }^{12}$, A. Davour ${ }^{19}$, C. T. Day ${ }^{13}$, C. De Clercq ${ }^{9}$, L. Demirörs ${ }^{8}$, F. Descamps ${ }^{22}$, P. Desiati ${ }^{5}$, T. DeYoung ${ }^{6}$, J. C. Diaz-Velez ${ }^{5}$, J. Dreyer ${ }^{10}$, J. P. Dumm ${ }^{5}$, M. R. Duvoort ${ }^{1}$, W. R. Edwards ${ }^{13}$, R. Ehrlich ${ }^{16}$, J. Eisch ${ }^{23}$, R. W. Ellsworth ${ }^{16}$, P. A. Evenson ${ }^{8}$, O. Fadiran ${ }^{24}$, A. R. Fazely ${ }^{25}$,

K. Filimonov ${ }^{12}$, M. M. Foerster ${ }^{6}$, B. D. Fox ${ }^{6}$, A. Franckowiak ${ }^{14}$ T. K. Gaisser ${ }^{8}$,

J. Gallagher ${ }^{26}$, R. Ganugapati ${ }^{5}$, H. Geenen ${ }^{14}$, L. Gerhardt ${ }^{11}$, A. Goldschmidtt ${ }^{13}$, J. A. Goodman ${ }^{16}$, R. Gozzini ${ }^{4}$, T. Griesel ${ }^{4}$, A. Groß $\aleph^{27}$, S. Grullon ${ }^{5}$, R. M. Gunasingha ${ }^{25}$, M. Gurtner ${ }^{14}$, A. Hallgren ${ }^{19}$, F. Halzen ${ }^{5}$, K. $\operatorname{Han}^{3}$, K. Hanson ${ }^{5}$, D. Hardtke ${ }^{12}$, R. Hardtke ${ }^{23}$, J. E. Hart ${ }^{6}$, Y. Hasegawa ${ }^{30}$, T. Hauschildt ${ }^{8}$, D. Hays ${ }^{13}$, J. Heise ${ }^{1}$, K. Helbing ${ }^{14}$, M. Hellwig ${ }^{4}$, P. Herquet ${ }^{20}$, G. C. Hill ${ }^{5}$, J. Hodges ${ }^{5}$, K. D. Hoffman ${ }^{16}$, B. Hommez ${ }^{22}$, K. Hoshina ${ }^{5}$, D. Hubert ${ }^{9}$, B. Hughey ${ }^{5}$, P. O. Hulth $^{18}$, J.-P. Hülß ${ }^{32}$, K. Hultqvist ${ }^{18}$, S. Hundertmark ${ }^{18}$, M. Inaba ${ }^{30}$, A. Ishihara ${ }^{5}$, J. Jacobsen ${ }^{13}$, G. S. Japaridze ${ }^{24}$, H. Johansson ${ }^{18}$, A. Jones ${ }^{13}$, J. M. Joseph ${ }^{13}$, K.-H. Kampert ${ }^{14}$, A. Kappes ${ }^{5,47}$, T. Karg ${ }^{14}$,

A. Karle ${ }^{5}$, H. Kawai ${ }^{30}$, J. L. Kelley ${ }^{5}$, N. Kitamura ${ }^{5}$, S. R. Klein ${ }^{13}$, S. Klepser ${ }^{2}$, G. Kohnen ${ }^{20}$, H. Kolanoski ${ }^{28}$, L. Köpke ${ }^{4}$, M. Kowalski ${ }^{28}$, T. Kowarik ${ }^{4}$, M. Krasberg ${ }^{5}$,

K. Kuehn ${ }^{11}$, M. Labare ${ }^{9}$, H. Landsman ${ }^{5}$, H. Leich ${ }^{2}$, D. Leier ${ }^{10}$, I. Liubarsky ${ }^{29}$, J. Lundberg ${ }^{19}$, J. Lünemann ${ }^{10}$, J. Madsen ${ }^{23}$, K. Mase ${ }^{30}$, H. S. Matis ${ }^{13}$, T. McCauley ${ }^{13}$, C. P. McParland ${ }^{13}$, A. Meli ${ }^{10}$, T. Messarius ${ }^{10}$, P. Mészáros ${ }^{6,21}$, H. Miyamoto ${ }^{30}$, A. Mokhtarani ${ }^{13}$, T. Montaruli ${ }^{5,35}$, A. Morey ${ }^{12}$, R. Morse $^{5}$, S. M. Movit ${ }^{21}$, K. Münich ${ }^{10}$, R. Nahnhauer ${ }^{2}$, J. W. Nam ${ }^{11}$, P. Nießen ${ }^{8}$, D. R. Nygren ${ }^{13}$, H. Ögelman ${ }^{5}$, A. Olivas ${ }^{16}$, S. Patton ${ }^{13}$, C. Peña-Garay ${ }^{7}$, C. Pérez de los $\operatorname{Heros}^{19}$, A. Piegsa ${ }^{4}$, D. Pieloth ${ }^{2}$, A. C. Pohl ${ }^{19,36}$, R. Porrata ${ }^{12}$, J. Pretz ${ }^{16}$, P. B. Price ${ }^{12}$, G. T. Przybylski ${ }^{13}$, K. Rawlins ${ }^{31}$, S. Razzaque ${ }^{6,21}$, E. Resconi ${ }^{27}$, W. Rhode ${ }^{10}$, M. Ribordy ${ }^{20}$, A. Rizzo ${ }^{9}$, S. Robbins ${ }^{14}$, P. Roth $^{16}$, C. Rott ${ }^{6}$, D. Rutledge ${ }^{6}$, D. Ryckbosch ${ }^{22}$, H.-G. Sander ${ }^{4}$, S. Sarkar ${ }^{33}$, S. Schlenstedt ${ }^{2}$, T. Schmidt ${ }^{16}$, D. Schneider ${ }^{5}$, D. Seckel ${ }^{8}$, B. Semburg ${ }^{14}$, S. H. Seo ${ }^{6}$, S. Seunarine ${ }^{3}$, A. Silvestri ${ }^{11}$, A. J. Smith ${ }^{16}$, M. Solarz ${ }^{12}$, C. Song ${ }^{5}$, J. E. Sopher ${ }^{13}$, G. M. Spiczak ${ }^{23}$, C. Spiering ${ }^{2}$, M. Stamatikos ${ }^{5,38}$, T. Stanev ${ }^{8}$, P. Steffen ${ }^{2}$, T. Stezelberger ${ }^{13}$,

R. G. Stokstad ${ }^{13}$, M. C. Stoufer ${ }^{13}$, S. Stoyanov ${ }^{8}$, E. A. Strahler ${ }^{5}$, T. Straszheim ${ }^{16}$, K.-H. Sulanke ${ }^{2}$, G. W. Sullivan ${ }^{16}$, T. J. Sumner ${ }^{29}$, I. Taboada ${ }^{12}$, O. Tarasova ${ }^{2}$, A. Tepe ${ }^{14}$, L. Thollander ${ }^{18}$, S. Tilav ${ }^{8}$, M. Tluczykont ${ }^{2}$, P. A. Toale ${ }^{6}$, D. Turčan ${ }^{16}$, N. van Eijndhoven ${ }^{1}$, 
This is an unedited preprint of an article accepted for publication in The Astrophysical Journal. The final published article may differ from this preprint. Copyright 2007 by The American Astronomical Society. Please cite as 'ApJ preprint doi:10.1086/'524920".

J. Vandenbroucke ${ }^{12}$, A. Van Overloop ${ }^{22}$, V. Viscomi ${ }^{6}$, B. Voigt $^{2}$, W. Wagner ${ }^{10}$, C. Walck ${ }^{18}$, H. Waldmann ${ }^{2}$, M. Walter ${ }^{2}$, Y.-R. Wang ${ }^{5}$, C. Wendt ${ }^{5}$, C. H. Wiebusch ${ }^{32}$, G. Wikström ${ }^{18}$, D. R. Williams ${ }^{6}$, R. Wischnewski ${ }^{2}$, H. Wissing ${ }^{32}$, K. Woschnagg ${ }^{12}$, X. W. Xu ${ }^{25}$, G. Yodh ${ }^{11}$, S. Yoshida ${ }^{30}$, J. D. Zornoza ${ }^{5,37}$ (the IceCube Collaboration), and M. Boer ${ }^{39}$, T. Cline ${ }^{38}$, G. Crew ${ }^{40}$, M. Feroci ${ }^{41}$, F. Frontera ${ }^{42}$, K. Hurley ${ }^{43}$, D. Lamb ${ }^{44}$, A. Rau ${ }^{45}$, F. Rossi ${ }^{42}$, G. Ricker ${ }^{40}$, A. von Kienlin ${ }^{46}$ (the InterPlanetary Network) 
This is an unedited preprint of an article accepted for publication in The Astrophysical Journal. The final published article may differ from this preprint. Copyright 2007 by The American Astronomical Society. Please cite as 'ApJ preprint doi:10.1086/'524920".

${ }^{1}$ Dept. of Physics and Astronomy, Utrecht University/SRON, NL-3584 CC Utrecht, The Netherlands

${ }^{2}$ DESY, D-15735 Zeuthen, Germany

${ }^{3}$ Dept. of Physics and Astronomy, University of Canterbury, Private Bag 4800, Christchurch, New Zealand

${ }^{4}$ Institute of Physics, University of Mainz, Staudinger Weg 7, D-55099 Mainz, Germany

${ }^{5}$ Dept. of Physics, University of Wisconsin, Madison, WI 53706, USA

${ }^{6}$ Dept. of Physics, Pennsylvania State University, University Park, PA 16802, USA

${ }^{7}$ Institute for Advanced Study, Princeton, NJ 08540, USA

${ }^{8}$ Bartol Research Institute, University of Delaware, Newark, DE 19716, USA

${ }^{9}$ Vrije Universiteit Brussel, Dienst ELEM, B-1050 Brussels, Belgium

${ }^{10}$ Dept. of Physics, Universität Dortmund, D-44221 Dortmund, Germany

${ }^{11}$ Dept. of Physics and Astronomy, University of California, Irvine, CA 92697, USA

${ }^{12}$ Dept. of Physics, University of California, Berkeley, CA 94720, USA

${ }^{13}$ Lawrence Berkeley National Laboratory, Berkeley, CA 94720, USA

${ }^{14}$ Dept. of Physics, University of Wuppertal, D-42119 Wuppertal, Germany

${ }^{15}$ Université Libre de Bruxelles, Science Faculty CP230, B-1050 Brussels, Belgium

${ }^{16}$ Dept. of Physics, University of Maryland, College Park, MD 20742, USA

${ }^{17}$ Dept. of Physics and Astronomy, University of Kansas, Lawrence, KS 66045, USA

${ }^{18}$ Dept. of Physics, Stockholm University, SE-10691 Stockholm, Sweden

${ }^{19}$ Division of High Energy Physics, Uppsala University, S-75121 Uppsala, Sweden

${ }^{20}$ University of Mons-Hainaut, 7000 Mons, Belgium

${ }^{21}$ Dept. of Astronomy and Astrophysics, Pennsylvania State University, University Park, PA 16802, USA

${ }^{22}$ Dept. of Subatomic and Radiation Physics, University of Gent, B-9000 Gent, Belgium

${ }^{23}$ Dept. of Physics, University of Wisconsin, River Falls, WI 54022, USA

${ }^{24}$ CTSPS, Clark-Atlanta University, Atlanta, GA 30314, USA

${ }^{25}$ Dept. of Physics, Southern University, Baton Rouge, LA 70813, USA

${ }^{26}$ Dept. of Astronomy, University of Wisconsin, Madison, WI 53706, USA

${ }^{27}$ Max-Planck-Institut für Kernphysik, D-69177 Heidelberg, Germany

${ }^{28}$ Institut für Physik, Humboldt Universität zu Berlin, D-12489 Berlin, Germany

${ }^{29}$ Blackett Laboratory, Imperial College, London SW7 2BW, UK

${ }^{30}$ Dept. of Physics, Chiba University, Chiba 263-8522 Japan 
This is an unedited preprint of an article accepted for publication in The Astrophysical Journal. The final published article may differ from this preprint. Copyright 2007 by The American Astronomical Society. Please cite as 'ApJ preprint doi:10.1086/'524920".

* Corresponding author K. Kuehn: kuehn@HEP.ps.uci.edu

\begin{abstract}
We present the results of the analysis of neutrino observations by the Antarctic Muon and Neutrino Detector Array (AMANDA) correlated with photon observations of more than 400 gamma-ray bursts (GRBs) in the Northern Hemisphere from 1997 to 2003. During this time period, AMANDA's effective collection area for muon neutrinos was larger than that of any other existing detector. After the application of various selection criteria to our data, we expect $\sim 1$ neutrino event and $<2$ background events. Based on our observations of zero events during and immediately prior to the GRBs in the dataset, we set the most stringent upper
\end{abstract}

\footnotetext{
${ }^{31}$ Dept. of Physics and Astronomy, University of Alaska Anchorage, 3211 Providence Dr., Anchorage, AK 99508, USA

${ }^{32}$ III Physikalisches Institut, RWTH Aachen University, D-52074 Aachen, Germany

${ }^{33}$ Dept. of Physics, University of Oxford, 1 Keble Road, Oxford OX1 3NP, UK

${ }^{34}$ Deceased

${ }^{35}$ on leave of absence from Università di Bari, Dipartimento di Fisica, I-70126, Bari, Italy

${ }^{36}$ affiliated with Dept. of Chemistry and Biomedical Sciences, Kalmar University, S-39182 Kalmar, Sweden

${ }^{37}$ affiliated with IFIC (CSIC-Universitat de València), A. C. 22085, 46071 Valencia, Spain

${ }^{38}$ NASA Goddard Space Flight Center, Greenbelt, MD 20771, USA

${ }^{39}$ Observatoire de Haute Provence, F-04870 Saint Michel l'Observatoire, France

${ }^{40}$ Massachusetts Institute of Technology, Kavli Institute for Astrophysics and Space Research, Cambridge, MA 02139, USA

${ }^{41}$ Instituto di Astrofisica Spaziale e Fisica Cosmica, Instituto Nazionale di Astrofisica, c/o CNR Area di Ricerca di Roma - Tor Vergata, Via Fosso del Cavaliere 100, Roma, I-00133 Italy

${ }^{42}$ Physics Department, University of Ferrara, Via Saragat 1, 44100 Ferrara, Italy

${ }^{43}$ University of California, Space Sciences Laboratory, 7 Gauss Way, Berkeley, CA 94720-7450 USA

${ }^{44}$ Department of Astronomy and Astrophysics, University of Chicago, 5640 South Ellis Avenue Chicago, IL 60637 USA

${ }^{45}$ Caltech Optical Observatories, California Institute of Technology, MS 105-24, 1200 E. California Blvd., Pasadena, CA 91125 USA

${ }^{46}$ Max-Planck-Institut für Extraterrestrische Physik, Giessenbachstrasse, 85748, Garching, Germany

${ }^{47}$ on leave of absence from Universität Erlangen-Nürnberg, D-91058, Erlangen, Germany
} 
This is an unedited preprint of an article accepted for publication in The Astrophysical Journal. The final published article may differ from this preprint. Copyright 2007 by The American Astronomical Society. Please cite as 'ApJ preprint doi:10.1086/'524920".

limit on muon neutrino emission correlated with gamma-ray bursts. Assuming a Waxman-Bahcall spectrum and incorporating all systematic uncertainties, our flux upper limit has a normalization at $1 \mathrm{PeV}$ of $E^{2} \Phi_{\nu} \leq 6.3 \times 10^{-9} \mathrm{GeVcm}^{-2} \mathrm{~s}^{-1} \mathrm{sr}^{-1}$, with $90 \%$ of the events expected within the energy range of $\sim 10 \mathrm{TeV}$ to $\sim 3 \mathrm{PeV}$. The impact of this limit on several theoretical models of GRBs is discussed, as well as the future potential for detection of GRBs by next generation neutrino telescopes. Finally, we briefly describe several modifications to this analysis in order to apply it to other types of transient point sources.

Subject headings: gamma-ray bursts, high energy astrophysics, neutrino astronomy, AMANDA

\section{Introduction}

\subsection{Gamma-Ray Bursts}

Gamma-ray bursts (GRBs) are among the most energetic phenomena in the universe; based on their luminosity and the cosmological distances derived from redshift measurements of burst afterglows and/or host galaxies (Costa et al. 2003), GRBs require the release of an enormous amount of energy $\left(\mathrm{E} \approx 10^{53} \times \Omega / 4 \pi \mathrm{erg}\right.$, where $\Omega$ is the solid angle of the GRB jet) in as little as a fraction of a second (Frail et al. 2001). Based on the observations of the Burst and Transient Source Experiment (BATSE, see Fishman et al. 1993) and other space-based detectors, they are expected to occur throughout the observable universe at a rate of $\gtrsim 700$ per year, though current instrument do not have sufficient sky coverage or sensitivity to detect every burst. Long duration ( $\gtrsim 2 \mathrm{sec}$ ) bursts are believed to originate from the collapse of a massive stellar progenitor into a black hole (Woosley 1993; Paczyński 1998), whereas short duration $(\lesssim 2 \mathrm{sec})$ bursts are believed to result from the merger of two compact objects into a black hole (Eicher et al. 1989) ${ }^{1}$. Though these two types of bursts come from different progenitors, both are consistent with the canonical picture of gamma-ray bursts - the fireball scenario (Paczyński 1986; Goodman et al. 1986).

A fireball is generated during the formation of the black hole when the outflowing plasma is accelerated to ultra-relativistic speeds. Additionally, baryonic matter from the progenitor

\footnotetext{
${ }^{1}$ For a more recent treatment of the compact object merger scenario, see (Paczyński 1998b; Lewin et al. 2006), and for an alternative description of the GRB progenitor scenario, see also (Roming et al. 2006).
} 
This is an unedited preprint of an article accepted for publication in The Astrophysical Journal. The final published article may differ from this preprint. Copyright 2007 by The American Astronomical Society. Please cite as 'ApJ preprint doi:10.1086/'524920".

is expected to be entrained with the plasma as it is accelerated. The amount of matter swept up by the fireball will be proportional to the total energy of the fireball:

$$
M_{\text {baryons }}=E_{\text {fireball }} / \Gamma c^{2}
$$

where $\Gamma$ is the Lorentz boost factor and $\mathrm{c}$ is the speed of light. Subsequently, in an optically thin region (outside of the progenitor), the kinetic energy of the plasma is converted to radiation, either through interaction with an external medium or through self-interaction within the flow (Narayan 1992; Rees 1992). Photo-pion production by baryon interaction with synchrotron or inverse Compton scattered photons will then lead to several decay products, including neutrinos and antineutrinos in a ratio of 2:1 (Waxman \& Bahcall 1997). The primary reaction is:

$$
p+\gamma \rightarrow \Delta \rightarrow \pi^{+}+n
$$

followed by

$$
\pi^{+} \rightarrow \mu^{+}+\nu_{\mu}
$$

after which the muon will further decay to

$$
\mu^{+} \rightarrow e^{+}+\nu_{e}+\overline{\nu_{\mu}}
$$

Similarly, "precursor" neutrinos may be generated by p-p interactions either within the star or in the immediate circumburst environment (see Section 2).

Alternate mechanisms for GRB emission, such as that responsible for "Poynting fluxdriven" bursts (Lyutikov \& Blandford 2003), are based upon electromagnetic rather than hydrodynamic sources of energy to power the burst. These mechanisms represent a significant departure from the fireball scenario described above, though they are still consistent with all photon observations of GRBs to date. However, a distinguishing characteristic of these alternate mechanisms is that they do not lead to significant acceleration of baryons in the GRB. Equations (2)-(4), then, are not relevant for GRBs powered by the Poynting flux mechanism. As a result, the expected neutrino flux from such bursts is essentially zero. Therefore, the positive detection of GRB neutrinos is one way to clearly distinguish the fireball mechanism from the Poynting flux mechanism of GRB production.

Due to their minuscule interaction cross section (Gandhi et al. 1998), any neutrinos that are produced by a GRB will reach the AMANDA detector after traveling nearly unimpeded from the GRB environment. AMANDA has been searching for high-energy neutrinos from various astrophysical fluxes (both discrete and diffuse) for nearly a decade; in this work we focus on the analysis of AMANDA data correlated with photon observations of more than 400 GRBs from 1997 to 2003. 
This is an unedited preprint of an article accepted for publication in The Astrophysical Journal. The final published article may differ from this preprint. Copyright 2007 by The American Astronomical Society. Please cite as 'ApJ preprint doi:10.1086/'524920".

\subsection{The AMANDA Detector}

The AMANDA detector (Ahrens et al. 2002) is an array of Optical Modules (OMs) deployed at depths between 1.5 and $2 \mathrm{~km}$ beneath the surface of the ice at the South Pole. An $\mathrm{OM}$ consists of a photomultiplier tube housed in a glass pressure sphere. During the years 1997-1999 the detector operated with 302 OMs on ten strings placed in a circular geometry with a diameter of about $100 \mathrm{~m}$, and was known as AMANDA B-10. From 2000 onward, nine additional strings were in operation, placed within a diameter of about $200 \mathrm{~m}$, bringing the total number of optical modules to 677 . This phase of the neutrino observatory (dubbed AMANDA-II) operated through 2004, and continues as a high density component of IceCube, a km-scale detector currently being constructed (Achterberg et al. 2006a).

The optical modules in AMANDA are designed to detect the Cherenkov emission from neutrino-induced muons that travel through or near the instrumented volume of ice. While other neutrinos may be detected with this search, the efficiency for $\nu_{e}$ or $\nu_{\tau}$ detection is significantly smaller. Other multi-flavor GRB neutrino searches which don't require directional information have been performed (Achterberg et al. 2007); we focus here on the search for GRB muon neutrinos from the Northern Hemisphere $\left(\delta\right.$ from $0^{\circ}$ to $\left.90^{\circ}\right)$. Due to the limited volume of ice above the detector, few downgoing extraterrestrial neutrinos will interact above and be detected by AMANDA. At the energies of interest to this analysis, the down-going events in the AMANDA dataset are primarily the atmospheric muon background which will completely overwhelm any potential downgoing signal. Thus, our extraterrestrial signal is primarily confined to the horizontal or up-going direction. As these muon neutrinos travel through the ice, they may interact with nearby nucleons to create energetic muons:

$$
\nu_{\mu}+N \rightarrow \mu+X
$$

where $\mathrm{N}$ is a nucleon and $\mathrm{X}$ represents other reaction products. Muons produced in this reaction can carry a significant fraction of the original neutrino energy (Gandhi et al. 1996). Depending on its energy, the muon can travel up to tens of kilometers through the ice; for $\nu_{\mu}$ in the energy range of greatest interest to AMANDA $\left(\sim 10^{5} \mathrm{GeV}\right)$, the muon path length is $\sim 10 \mathrm{~km}$ (Lipari \& Stanev 1991).

Since AMANDA can detect such a muon anywhere along its substantial path length, the effective detector volume is significantly larger than the actual instrumented volume. A muon that has sufficient energy will continuously emit Cherenkov radiation, and will also generate additional particles due to stochastic processes. The ice at a depth of more than one kilometer is extremely clear, and thus the Cherenkov photons have large scattering $\left(L_{\mathrm{s}}^{\mathrm{eff}}\right)$ and absorption $\left(L_{\mathrm{a}}\right)$ lengths - at $\lambda=400 \mathrm{~nm}, L_{\mathrm{s}}^{\mathrm{eff}} \approx 25 \mathrm{~m}$ and $L_{\mathrm{a}} \approx 100 \mathrm{~m}$ (Ackermann et al. 2006). The Cherenkov light therefore has the potential to reach numerous OMs as the muon travels 
This is an unedited preprint of an article accepted for publication in The Astrophysical Journal. The final published article may differ from this preprint. Copyright 2007 by The American Astronomical Society. Please cite as 'ApJ preprint doi:10.1086/'524920".

through the detector, and the relative timing of the hit OMs provides the basis for a set of maximum-likelihood reconstruction algorithms to determine the muon's direction of origin (Ahrens et al. 2004). The algorithms applied to this analysis are based on variations from a randomly-seeded "first guess" track using the Pandel function to parametrize the sequence of OM hits. The likelihood of the initial track is calculated, and then the procedure is iterated (up to 32 times) to determine the most likely muon track. Iterations beyond the first incorporate increasingly complex features of the detector response to the Cherenkov photons, the details of which are beyond the scope of this work ${ }^{2}$. Detector simulations, along with observations of downgoing cosmic ray muons, have shown that this procedure provides track reconstructions accurate to within a mean value of $\sim 2^{\circ}$. Atmospheric muons are almost entirely removed from the dataset by constraining our search to those bursts occurring in the Northern Hemisphere, allowing the detector to be shielded from a substantial background flux by the bulk of the earth. Upgoing atmospheric neutrinos caused by cosmic ray interactions in the Northern hemisphere may also be detected by AMANDA, as their spectrum extends into the energy range of relevance to the GRB search. However, they likewise are removed from the dataset by requiring strict spatial and temporal correlation with photon observations of GRBs. With these selection criteria applied, we expect less than 0.01 atmospheric neutrino events in our dataset.

In Section 2 we describe several models for GRB neutrino emission. In Section 3 we discuss the method for determining periods of stable detector performance and for separating the expected GRB neutrino signal from all misreconstructed background events, as well as the systematic uncertainties associated with this analysis procedure. In Section 4 we compare the results of the AMANDA observations with the models, as well as provide a spectrumindependent method for determining the fluence upper limit from GRBs. We conclude with the future potential of AMANDA/IceCube, for both the standard GRB search in the Swift era (Markwardt et al. 2005) and for searches optimized for other transient point sources, such as jet-driven supernovae.

\section{Models of Neutrino Emission}

According to the canonical description provided above, gamma-ray bursts result from the dissipation of the energy of relativistic outflows from a central engine. Based on the assumption that GRBs are the source of ultra-high energy cosmic rays (UHECRs), Waxman

\footnotetext{
${ }^{2}$ An alternative track reconstruction known as a "paraboloid fit" is also relevant for our secondary data selection criteria, see Section 3.3 for further details.
} 
This is an unedited preprint of an article accepted for publication in The Astrophysical Journal. The final published article may differ from this preprint. Copyright 2007 by The American Astronomical Society. Please cite as 'ApJ preprint doi:10.1086/'524920".

and Bahcall predicted an annual muon neutrino flux associated with GRBs of $E^{2} \Phi_{\nu} \sim 9$ $\times 10^{-9} \mathrm{GeVcm}^{-2} \mathrm{~s}^{-1} \mathrm{sr}^{-1}$ from $100 \mathrm{TeV}$ to $10 \mathrm{PeV}$ (Waxman 2003) ${ }^{3}$. Murase \& Nagataki (2006a) predict a similar spectrum to Waxman and Bahcall for long-duration bursts, though their simulations include a wider range of parameters, leading to a wider variation in predicted fluxes. Inclusion of neutrino oscillations reduce these predictions by a factor of two ${ }^{4}$. Additional models requiring more specific assumptions about the nature of GRBs have also been proposed. Razzaque et al. (2003a) hypothesize a scenario in which a supernova precedes a long-duration GRB by several days to a week. In this "supranova" scenario, the supernova remnant provides target nucleons for pp interactions leading to precursor neutrinos with energy $E_{\nu} \sim 10 \mathrm{TeV}$. Furthermore, the remnant will produce target photons for $\mathrm{p} \gamma$ interactions, which will also yield muon neutrinos up to $10^{16} \mathrm{eV}$, albeit with a different spectral shape than that predicted by the Waxman-Bahcall model ${ }^{5}$. This model also has implications for gamma-ray dark (or "choked") bursts, which are briefly discussed in Section 5. Furthermore, though a number of models explicitly incorporate only long-duration bursts into their models, the GRB central engine is in principle independent of burst type. Thus, though the flux upper limits for these models include long bursts only (except, importantly, for the model of Waxman \& Bahcall), the models could potentially be expanded to include neutrinos from short bursts as well. Within the AMANDA dataset long bursts dominate over short bursts; incorporating short bursts would have a small, though not insignificant, effect on the overall limit (see Section 4 for details). Figure 1 shows the expected GRB neutrino flux based on four representative models. The precursor model predicts a neutrino flux as early as several tens of seconds prior to the observed GRB photons, whereas the other models tested here predict a neutrino flux in coincidence with the GRB photons ${ }^{6}$. Other models of GRB emission also exist (see, e.g. Dermer \& Atoyan 2006, 2003); though we do not explicitly focus on such models here, a flux upper limit can be calculated for such models using the Green's Function method detailed in Section 4.

\footnotetext{
${ }^{3}$ For the original formulation of this neutrino flux prediction, see (Waxman \& Bahcall 1997). Note that this GRB neutrino flux is distinct from the Waxman-Bahcall upper bound on the diffuse neutrino flux due to UHECRs.

${ }^{4}$ Oscillations modify the flavor ratio from 1:2:0 at the source to 1:1:1 at Earth. However, see (Kashti \& Waxman 2005) for a discussion regarding different flavor ratios due to energy losses of the $\pi$ and $\nu$.

${ }^{5}$ Though the supranova model is still within the realm of possibility, it is somewhat disfavored based on observations of GRB060218, in which the supernova preceded the GRB by at most a few hours - not long enough to provide an ideal circumburst environment for a significant neutrino flux.

${ }^{6}$ Any time delay in observing the neutrinos due to the neutrino mass is assumed to be negligible compared to the time scale over which we search for the burst emission.
} 
This is an unedited preprint of an article accepted for publication in The Astrophysical Journal. The final published article may differ from this preprint. Copyright 2007 by The American Astronomical Society. Please cite as 'ApJ preprint doi:10.1086/'524920".

Many theoretical models (most notably, the Waxman-Bahcall model) are based on assumptions regarding the burst environment as well as the average properties of bursts (total emission energy, redshift, etc.) which do not correspond directly to the properties of specific bursts. It is possible to estimate the muon neutrino flux for individual bursts, but these estimates vary substantially, and often bracket the predictions of the averaged properties (Stamatikos 2005). For those bursts where redshift and spectral information is available, more accurate estimates of muon neutrino flux can be made on a burst-by-burst basis. For extremely bright, nearby bursts (e.g. GRB030329), the predicted fluxes can be as much as two orders of magnitude greater than the mean burst flux (Stamatikos 2006). Finally, our simulations assume a $\Phi_{\nu}: \Phi_{\bar{\nu}}$ ratio of 1:1. AMANDA does not distinguish the muon charge; however, neutrino event rates are larger than anti-neutrino rates for an equal flux, since the neutrino cross section is larger up to energies of $\sim 10^{5} \mathrm{GeV}$. Thus, any models proposing a ratio other than unity will result in a different expected event rate and, ultimately, a different flux upper limit for this analysis.

\section{Observation Procedure}

\subsection{Correlated Observations}

This AMANDA GRB search relies on spatial and temporal correlations with photon observations of other instruments including BATSE aboard the Compton Gamma-Ray Observatory (CGRO), as well as HETE-II, Ulysses, and other satellites of the Third Interplanetary Network (IPN) (Hurley 1998). As stated previously, our search is restricted to that half of the bursts occurring in the Northern Hemisphere. Furthermore, because engineering and maintenance work is performed on the AMANDA detector during the austral summer (December-February), only a few bursts from these months can potentially be observed each year. For each GRB in the dataset, we search for muon neutrino emission during the coincident phase of burst emission. The coincident phase is determined by either the $\mathrm{T}_{90}$ start and end times of the burst, or the entire duration of emission in excess of the background rate (for bursts without well-defined $\left.\mathrm{T}_{90}\right)$. A period of time before and after each burst is added to the search in order to accommodate the timing errors of the photon observations (which vary from burst to burst). Most bursts have prompt phases lasting from a few seconds up to to a few tens of seconds, though there are some exceptional bursts lasting hundreds of seconds. To investigate different model predictions for the bursts occurring during 2001-2003, we also performed an extended search for precursor neutrinos from 110 seconds before the burst start time until the beginning of the coincident search window. BATSE observations were the sole source of data for the AMANDA B-10 analysis for 1997-1999. Other IPN-detected 
This is an unedited preprint of an article accepted for publication in The Astrophysical Journal. The final published article may differ from this preprint. Copyright 2007 by The American Astronomical Society. Please cite as 'ApJ preprint doi:10.1086/'524920".

bursts were included beginning with the AMANDA-II dataset in 2000, and the analysis then relied exclusively on IPN data from other satellites once CGRO was decommissioned in May, 2000. Additional bursts were also discovered in the BATSE archival data (Kommers 1998; Stern et al. 2001); the relevant time periods of the AMANDA data were searched for muon neutrinos from these bursts as well. We do not, however, include this particular subset of bursts in the flux or fluence upper limits for the models addressed in this work, because non-triggered bursts were not incorporated into the primary models of GRB neutrino emission. The instruments participating in the Interplanetary Network through 2003 are given in Table 1 and the number of bursts searched in each year of AMANDA observations is listed in Table 2; information on the specific bursts included in this analysis is also available (Kuehn 2007).

\subsection{Background and Detector Stability}

To determine the background rate and to establish data selection criteria for each burst, a larger period of one hour and 50 minutes of data is analyzed - from one hour before the burst to one hour after the burst, with the 10 minute period during and immediately surrounding the burst excluded to ensure that the data quality cuts are not determined in a biased fashion (a "blind" analysis). Prior to determination of the data selection criteria, we study detector stability in this background period. The specific stability criteria for AMANDA B-10 have been discussed previously (Hardtke 2002); here we describe the AMANDA-II stability criteria in more detail.

We perform two tests to identify non-statistical fluctuations in the data rate that could produce fake events ("false positives") or unanticipated dead time ("false negatives") in the detector. The first test compares the observed event count per 10 second time bin to the expected, temporally uncorrelated, distribution of background events. This tests for any nonstatistical fluctuations in data rate due to temporary instability in the detector. Without this test, an upward fluctuation in the data rate not caused by neutrinos could potentially be misinterpreted as a signal event. This test has three successive steps based on the Pvalue of the event rate distribution. The $\mathrm{P}$-value of a data segment is defined as the percent difference between the RMS variation of the data event rate and the width of a Gaussian fit to the data rate distribution. The first step identifies all those bursts with stable periodsthose having a $\mathrm{P}$-value of less than $6 \%$ (corresponding to variations of less than $1 \sigma$ relative to the overall distribution of P-values). The second step identifies bursts with marginally stable detector performance: $6 \% \leq \mathrm{P} \leq 12 \%(1-2 \sigma)$. Additional tests are performed on these bursts; specifically, the data rate of the previously blinded 10-minute period is explored in a 
This is an unedited preprint of an article accepted for publication in The Astrophysical Journal. The final published article may differ from this preprint. Copyright 2007 by The American Astronomical Society. Please cite as 'ApJ preprint doi:10.1086/'524920".

region of the sky far away from the GRB (the "on-time, off-source" region). This maintains the blindness of the analysis, while allowing a more detailed exploration of detector stability. Marginally stable burst periods are included in the analysis if they are also marginally stable in the on-time, off-source region (P-value less than 12\%), and if the event rate has only small $(\leq 3 \sigma)$ variations throughout the on-time, off-source region. The vast majority of all burst time periods were stable according to these criteria. The final step of this test is applied if the first two steps are inconclusive. It requires any event rate variations greater than $3 \sigma$ to occur at a significant distance from the burst time. Two bursts fall into this category; they had marginally stable off-time periods and insufficient statistics for an on-time/off-source stability test. However they were included in this analysis because the largest event rate variations were separated in time from the burst by several minutes. Only one time period associated with a burst in the AMANDA dataset had off-time and on-time/off-source Pvalues greater than $12 \%$, and this burst was excluded from the analysis. Figure 2 shows the data rate per 10 seconds for a sample GRB period, overlaid with the Gaussian fit. They are in very good agreement, showing a stable data rate for this period of detector activity.

The second test utilizes the time between subsequent events $(\delta \mathrm{t})$ to ensure that there is not an anomalously large amount of time between detector triggers. The amount of time between triggers can vary widely, but larger gaps occur with much less frequency than shorter gaps. There is also unavoidable (but quantifiable) dead time between each trigger while the detector is being read out. The overall effect of the expected dead time is to reduce the detector's signal acceptance by approximately 17\%, and this quantity has been incorporated into the expected neutrino observation rate for this analysis. However, large unexpected gaps between triggers would indicate a period of unstable detector performance, and would mean that an otherwise detectable neutrino signal might not be observed during such a period. We test the 1 hour and 50 minute time periods surrounding each burst to ensure that no such gaps occur. An example of the temporal distribution of triggers compared with an exponentially decreasing fit to the $\delta$ t distribution is shown in Figure 3 . The variations observed in the data for this time period are within $2 \sigma$ of the observed fit for all values of $\delta$ t. Thus there are no unexpected variations in the time between detector triggers, and we confirm that AMANDA is collecting data as expected occurring during the on-time window for this burst. All data periods associated with GRBs that pass the first test also pass this second test for stable detector operation. 
This is an unedited preprint of an article accepted for publication in The Astrophysical Journal. The final published article may differ from this preprint. Copyright 2007 by The American Astronomical Society. Please cite as 'ApJ preprint doi:10.1086/'524920".

\subsection{Data Selection Criteria}

For those bursts determined to be stable by the above criteria, data quality cuts are then selected to separate the predicted signal from the observed background events. This process relies primarily on the simulated signal events and the observed background events. The simulation of the detector response to signal and background events is described in Ahrens et al. (Ahrens et al. 2004). The simulation procedure uses the neutrino generation program NuSim (Hill 1997) for signal event simulations. Background events are simulated with CORSIKA (Heck et al. 1998), which implements the 2001 version of the QGSJET model of hadronic interactions (Kalmykov \& Ostapchenko 1993). Once the neutrino or other cosmic-ray primaries are generated and propagated to their interaction vertex, we simulate the secondary propagation with the Muon Monte Carlo (MMC) package (Chirkin $\&$ Rhode 2004). Finally, we simulate the AMANDA detector response with the software package AMASIM. We then are able to compare simulated signal, simulated background, and observed background data.

In the case of the GRB search, the background rate is measured using the off-time window, where no signal is expected. Thus, unlike other AMANDA analyses (Ahrens et al. 2003a,b), the background events do not need to be simulated, nor do the data events need to be scrambled in time or azimuth to retain a blind analysis procedure. Exploring the variations between observed background events and simulated events does, however, ensure that we understand the systematic errors associated with the simulation process. For example, Figure 4 shows excellent agreement in $£_{\text {reco }}$, the $\log$ (Likelihood) of the reconstructed tracks of the simulated and observed background events. Given this level of agreement, the errors arising from discrepancies between the simulated and observed events are expected to be small. Additionally, atmospheric neutrinos have previously been observed by AMANDA up to $\mathrm{TeV}$ energies, and studies show that neutrinos from this proven source can be reconstructed with a high degree of accuracy(Andrés et al. 2001). Likewise, studies have been performed which compare simulated signal events with high-quality downgoing muon events (Hodges 2006). Because these downgoing events have similar properties to the simulated signal events, this provides additional assurance that the simulated signal events will have similar properties to the actual signal events we are attempting to observe. Section 3.4 gives a quantitative discussion of systematic errors.

To determine the set of data selection criteria that will produce the optimal flux upper limit in the absence of a signal, we minimize the Model Rejection Factor (MRF) (Hill \& Rawlins 2003). The MRF is based on the expected detector sensitivity prior to observations:

$$
M R F=\frac{\bar{\mu}_{90}\left(\mathrm{~N}_{\mathrm{BG}, \mathrm{Exp}}\right)}{\mathrm{N}_{\mathrm{Sig}}}
$$


This is an unedited preprint of an article accepted for publication in The Astrophysical Journal. The final published article may differ from this preprint. Copyright 2007 by The American Astronomical Society. Please cite as 'ApJ preprint doi:10.1086/'524920".

where $\bar{\mu}_{90}$ is the Feldman-Cousins $90 \%$ average event upper limit (Feldman \& Cousins 1998) derived from the expected number of background events $\left(\mathrm{N}_{\mathrm{BG} \text {,Exp }}\right)$ and $\mathrm{N}_{\mathrm{Sig}}$ is the expected number of signal events. $\mathrm{N}_{\mathrm{Sig}}$ is determined by convolving the theoretical spectrum $(\Phi=$ $\mathrm{dN}_{\nu} / \mathrm{dE}$ ) with the detector's energy- and angle-dependent effective neutrino collecting area $\left(A_{e f f, \nu}\right)$ and integrating over the angular acceptance of the detector, the energy range of interest $\left(10^{2}\right.$ to $10^{7} \mathrm{GeV}$ ), and the observation time (assuming 700 bursts contribute equally to the annual expected flux):

$$
N_{\mathrm{Sig}}=\iint \Phi(E, \theta, \phi) A_{\mathrm{eff}, \nu}(E, \theta) \mathrm{d} \mathrm{Ed} \Omega \mathrm{dt} .
$$

As an intermediate step in the determination of the expected number of signal events, we therefore need to determine the detector effective collection area. $A_{\mathrm{eff}, \nu}$ is determined by the fraction of simulated neutrino events that are retained after all data selection criteria are applied. This area also accounts for neutrinos that generate muons passing nearby (but not through) the detector and still cause the telescope to trigger.

In determining the optimal data selection criteria for the coincident search, we assume a Waxman-Bahcall neutrino spectrum (Waxman 2003); for the precursor search, we assume a Razzaque spectrum (Razzaque et al. 2003a). In addition to temporal coincidence described previously, the most relevant selection criterion for this analysis is the angular mismatch $\left(\Delta \Psi_{\mathrm{i}}\right)$ between the burst position and the reconstructed event track. This mismatch is determined for each of four separate maximum-likelihood pattern recognition algorithms (i $=1$ to 4) applied to the timing of the hit OMs (as described in Section 2). The different algorithms are based on different initial seeds and apply a different number of iterations to the track reconstruction procedure, thus they are able to provide different measures used for discrimination between expected signal and background events. Though they are not completely independent, they do offer improvements to the MRF when applied consecutively. The inherent difference in the muon and neutrino paths, as well as the inaccuracies of the reconstruction algorithms, prevent perfect characterization of all signal and background events. Nevertheless, the angular mismatch is quite effective as a selection criterion. For example, selecting events with a mismatch angle $\Delta \Psi_{1}$ of less than $12^{\circ}$ retains more than $90 \%$ of the expected signal events, while reducing the background to less than $0.5 \%$ (Figure 5 ). Depending upon the changes in the detector characteristics and the analysis tools from year to year, the MRF optimization procedure allowed for some variation in the specific track reconstruction algorithms applied, as well as the mismatch angle values selected for each algorithm (see Table 3).

Several secondary criteria were also used to improve the separation between signal and background events. Included in the secondary criteria is the measured number of hit channels - that is, the number of OMs participating in the reconstruction of each event. 
This is an unedited preprint of an article accepted for publication in The Astrophysical Journal. The final published article may differ from this preprint. Copyright 2007 by The American Astronomical Society. Please cite as 'ApJ preprint doi:10.1086/'524920".

The number of direct hits - hits that occur within $-15 /+75$ ns of the arrival time for light propagating from the reconstructed muon track to the $\mathrm{OM}$ in question-also serves as a useful criterion for data selection. Direct hits should be due to photons that do not scatter, or scatter minimally; their straight trajectories give them a well-defined behavior, making them most useful in determining the muon direction. Additionally, the likelihood of a given reconstruction and the angular resolution $\left(\sigma_{\Psi}\right)$ of the alternate event track reconstruction (the "paraboloid fit") provide a useful event discriminator, since high quality signal events will have higher likelihoods and superior angular resolution compared to the background events. One additional criterion used in this analysis is the uniformity of the spatial distribution of the hit OMs - events with hit OMs spread evenly along the track are more likely to be single high-energy neutrino-induced muons, whereas events with hit OMs clustered in time and space along the track are more likely to be background events. Different combinations of these criteria were applied in the 1997-1999, 2000, and 2001-2003 timeframes, as new analysis tools were developed and applied to the GRB neutrino search (see Table 3).

This analysis procedure was applied to bursts with localization errors from the satellite observations that are relatively small (typically less than $1^{\circ}$ ) and therefore inconsequential on the scale of the AMANDA search bin radius. However, several hundred IPN bursts have large localization errors ( $\gtrsim 1 / 2$ of the search bin radius), but still lie completely within the field of view of AMANDA. These were either marginal detections near the edge of BATSE's field of view or they were detected by only two IPN satellites, which prevents triangulation of their position but allows localization to an annular segment. Eleven of the bursts in the AMANDA dataset are only poorly localized; the increased search area for these bursts results in a corresponding increase in the expected background rate. To ensure that this increase does not diminish the overall sensitivity of the GRB search, more restrictive selection criteria are applied to these bursts. Whether well localized or poorly localized, each burst has an associated background expected during the burst time, calculated from the event rate of the off-time background region multiplied by the duration of the time window during which we search for signal events.

The initial criteria were independently selected to optimize the MRF and were then collectively optimized in an iterative fashion. The optimal criteria depended on the zenith angle of the burst, due to the higher observed background rate for bursts closer to the horizon. The criteria for higher background rates (i.e. low zenith angle bursts) were also applied to bursts with large satellite localization errors, regardless of the actual zenith angle of the burst. Table 3 lists all data selection criteria used for the year-by-year GRB analyses, as well as the selection criteria for the precursor search applied in 2001-2003. Though the data selection criteria are optimized for specific models of neutrino emission, other models can also be tested using the Green's Function Fluence Limit Method (see Results). While 
This is an unedited preprint of an article accepted for publication in The Astrophysical Journal. The final published article may differ from this preprint. Copyright 2007 by The American Astronomical Society. Please cite as 'ApJ preprint doi:10.1086/'524920".

the muon track reconstruction algorithm is very accurate, there is a small probability that a downgoing muon will be misreconstructed in the upgoing direction; such events are the primary background for the GRB search. After the application of data selection criteria, background events have an observed rate of $\sim 5 \times 10^{-5} \mathrm{~Hz}$ (with some seasonal variation), leading to a total of 1.74 expected background events over the entire observation period. Figure 6 show the effective area for neutrinos for the AMANDA-II detector after all data selection criteria are applied. Due to the large instrumented area and modest background rejection requirements of this analysis, AMANDA-II has an $A_{\text {eff }}$ significantly larger than any other contemporaneously-operating neutrino detector (e.g. Baikal (Spiering et al. 2004), SuperKamiokande (Fukuda et al. 2002), and SNO (Aharmim et al. 2000)). A determination of the relative MRF for a subset of bursts from the year 2000 analysis is shown in Figure 7 (the arrow indicates the MRF for the selected criteria). Using Equation 6, the number of expected GRB neutrino events for the Waxman-Bahcall model is shown in Table 3. We expect less than 1 event to occur in coincidence with all of the 419 bursts in the dataset.

Based on these values, we now calculate the flux sensitivity to simulated GRB neutrinos prior to "unblinding" the analysis and determining the number of events we actually observe. Results from the 268 bursts observed from 1997 to 1999 have been presented previously (Bay 2000; Hardtke 2002). We combine these initial observations with the results from the analysis of 151 bursts in the data collected in 2000-2003. The flux sensitivity for all 419 bursts is the MRF prior to observations (see Equation 5) multiplied by the normalization of the input spectrum; that is, $E^{2} \Phi_{\nu} \leq 2 \times 10^{-8} \mathrm{GeVcm}^{-2} \mathrm{~s}^{-1} \mathrm{sr}^{-1}$ for a Waxman-Bahcall muon neutrino spectrum with $90 \%$ of the events expected between $\sim 10 \mathrm{TeV}$ and $\sim 3 \mathrm{PeV}$. This sensitivity is calculated prior to the inclusion of systematic uncertainties.

\subsection{Uncertainties in Observation and Modeling}

There are several potential sources of systematic uncertainty in this analysis, including the Monte Carlo simulations of signal events, the modeling of the scattering and absorption lengths of the South Pole ice, and the OM response to incident photons. For the flux upper limits incorporating IPN bursts, the potential for inclusion of bursts which do not fit models based upon BATSE triggered bursts contributes to the overall uncertainty as well. Additionally, some bursts are of unknown duration-for the purposes of this search, they were classified as long-duration bursts so that we would not needlessly exclude any possible signal events. However, including all such bursts will potentially overestimate the signal event predictions for models based solely upon long-duration bursts. Finally, previous results from 1997-1999 were applied only to the Waxman-Bahcall model; limitations in the simulation 
This is an unedited preprint of an article accepted for publication in The Astrophysical Journal. The final published article may differ from this preprint. Copyright 2007 by The American Astronomical Society. Please cite as 'ApJ preprint doi:10.1086/'524920".

procedures in place at that time means that adapting these results to other models will introduce uncertainties in the expected neutrino event rate.

The scattering and absorption lengths of the ice were measured during the 1999-2000 austral summer with in situ lasers and LED flashers (Ackermann et al. 2006). While these measurements were extremely accurate, the limited precision with which they were implemented in our detector simulations contributes about $15 \%$ to the overall uncertainty. Furthermore, the quantum efficiency of the photomultiplier tubes is known to within $10 \%$, while the transmission efficiency of the glass pressure housing and the optical gel is known to a comparable precision. However, triggering depends on the detection of photons by 24 or more PMTs, so the uncertainty in a single OM does not translate directly into an uncertainty in the expected flux. Detailed simulations show that the quantum and transmission efficiencies together contribute only about $7 \%$ uncertainty in the expected neutrino flux (Ahrens et al. 2004). Though the GRB search implements a different methodology from other IceCube analyses (e.g. the point source search detailed in Achterberg et al. 2006b), the values for the individual contributions to the uncertainty are consistent across these different analyses.

Additionally, a statistical correction is required when IPN bursts are incorporated into the flux upper limits for models initially based on BATSE observations. In principle, BATSE has a sensitivity comparable to the suite of other IPN satellites treated collectively; observationally, their duration distribution seems qualitatively to be derived from the same bimodal population (Figure 8). However, the characteristics of the bursts detected by satellites with different sensitivities are not completely identical. BATSE non-triggered bursts have on average less than $1 / 10$ of the peak photon flux of their triggered counterparts, and if we assume that the neutrino flux scales as the photon flux, then including non-triggered bursts in the upper limit calculation would artificially increase the expected number of signal events, and thus lead to a flux upper limit that is too restrictive. We calculate (see Appendix A) that $12 \%$ of the IPN bursts should not be considered equivalent to BATSE triggered bursts, and thus should be excluded from the dataset. This leads to a $3 \%$ correction in the number of expected signal events. Furthermore, for models based solely on long-duration bursts such as (Murase \& Nagataki 2006a; Razzaque et al. 2003a), the inclusion of bursts of unknown duration may also lead to an overestimation of the number of expected signal events. In Appendix A, we derive a statistical correction of $6 \%$ to the expected number of signal events due to this effect.

Next, we determine the statistical correction required by the incorporation of position uncertainties in the BATSE GRB localizations. The mean position uncertainty for the 19972000 BATSE bursts is $4.1^{\circ}$, but this is skewed by a small number of bursts which have larger uncertainties (and which are not included in this analysis). The median position uncertainty 
This is an unedited preprint of an article accepted for publication in The Astrophysical Journal. The final published article may differ from this preprint. Copyright 2007 by The American Astronomical Society. Please cite as 'ApJ preprint doi:10.1086/'524920".

for 1997-2000 BATSE bursts is $3.2^{\circ}$; this is the quantity that is convolved with the AMANDA searchbin radius to determine the statistical correction to the AMANDA results. Placing each burst $3.2^{\circ}$ off-center in its searchbin is equivalent to shifting the searchbin radius by $3.2^{\circ}$ in either direction (see, e.g., Figure 5 , where this would result in a shift of the $\Delta \Psi$ acceptance region by 1-2 bins). A decrease in the searchbin radius reduces the signal acceptance in that direction, but that also necessitates an increase in the searchbin radius (and thus an increase in the signal acceptance) in the opposite direction. The majority of the expected signal is at very low $\Delta \Psi$; the decrease in the expected signal is therefore larger than the corresponding increase. Thus we determine that the BATSE position uncertainties will reduce the expected signal from the BATSE bursts by no more than $4 \%$. Because roughly $1 / 2$ of our total signal events are expected to come from the BATSE bursts, this results in a final statistical correction of only $2 \%$. It is important to note that the vast majority of the IPN bursts were observed with ground-based follow-up observations, thus their position uncertainties are on the order of arcminutes (or even arcseconds), which is very much smaller than the AMANDA searchbin and the resulting uncertainty in the AMANDA analysis can therefore be neglected.

Finally, we determine the uncertainty introduced when the previous results from 19971999 are applied to theoretical predictions other than the Waxman-Bahcall model. Though the uncertainties specifically for the Waxman-Bahcall model are well understood and are incorporated into the previous results, limitations in the simulation procedures at the time of the previous analysis lead to a further uncertainty in the neutrino event rate for the Murase-Nagataki and Razzaque et al. models of $\sim 20 \%$. When we combine the results from the 268 bursts from 1997-1999 with the results from 151 bursts from 2000-2003 into a single flux upper limit, we assume conservatively that the neutrino event rate for the bursts from 1997-1999 is overestimated by $20 \%$.

All significant sources of uncertainty for the GRB analysis, along with the correction factors, are summarized in Table 4. While the reduction in the expected neutrino event rate for the 1997-199 bursts is not specifically enumerated in this Table, it is incorporated into the relevant flux upper limits discussed in the next section. Assuming no correlation among the other uncertainties, we summed the different factors in quadrature and applied the other relevant corrections to obtain a total uncertainty of $+12 \% /-22 \%(+6 \% /-28 \%$ for models based on long-duration bursts only) in the total detector exposure, and therefore in the number of signal events and the flux and fluence upper limits. This is comparable to the uncertainty determined by (Hodges 2006), who also characterized the agreement between the simulated signal events and high-quality downgoing muon events, which served as a proxy for the expected signal events for AMANDA analyses. 
This is an unedited preprint of an article accepted for publication in The Astrophysical Journal. The final published article may differ from this preprint. Copyright 2007 by The American Astronomical Society. Please cite as 'ApJ preprint doi:10.1086/'524920".

\section{Results}

We observe zero events from the 419 Northern Hemisphere bursts searched during the years 1997 to 2003, which is consistent with the background estimate of 1.74 events (Table $5)^{7}$. Since the observed number of events is less than the expected background, the flux upper limits for the coincident muon neutrino search are nearly a factor of three better than the expected sensitivity (i.e. the observed MRF for a Waxman-Bahcall flux is 1.36 compared to the expected value of 3.80). Figure 9 shows the $90 \%$ C.L. flux upper limits relative to the Waxman-Bahcall, Razzaque, and Murase-Nagataki models. Though our analysis was restricted to bursts located in the Northern Hemisphere $(2 \pi \mathrm{sr})$, all flux upper limits are for the entire sky $(4 \pi \mathrm{sr})$. Including the systematic uncertainties in the manner outlined by Conrad et al. (2003), we calculate the coincident muon neutrino flux upper limit for the Waxman-Bahcall spectrum to have a normalization at $1 \mathrm{PeV}$ of

$E^{2} \Phi_{\nu} \leq 6.3 \times 10^{-9} \mathrm{GeVcm}^{-2} \mathrm{~s}^{-1} \mathrm{sr}^{-1}$

with $90 \%$ of the events expected between $\sim 10 \mathrm{TeV}$ and $\sim 3 \mathrm{PeV}$.

We place similar constraints on the model parameters of Murase \& Nagataki (2006a). Based on our null result, Parameter Set $\mathrm{C}$ is highly disfavored for all variations in their parameters, though this particular set is disfavored on other grounds as well, and is only briefly described in their work. Parameter Set $\mathrm{A}$ is ruled out $(\mathrm{MRF}=0.92)$ by the current AMANDA observations at the $90 \%$ confidence level. However, it is important to note that Parameter Set A uses a baryon loading factor that is fine-tuned to provide significant neutrino flux. Other, possibly more realistic, values for the baryon loading would significantly reduce the expected neutrino emission, and therefore result in an MRF that is higher by an order of magnitude or more. The original model incorporates only long-duration bursts that follow the cosmic star-formation rate (Murase \& Nagataki 2006c); incorporating all short bursts would yield flux upper limits that are better than those presented here by approximately $13 \%$, which includes removing the "correction" due to incorporating of bursts of unknown duration (see Section 3.4).

Our combined results from 1997-2003 also constrain the supranova model of Razzaque et al. We begin by considering the assumption that all GRBs are preceded by supernovae that produce a circumburst environment ideally suited for neutrino production. The observed MRF for this case is 0.45 , and thus we exclude the predicted neutrino flux at the $90 \%$ level.

\footnotetext{
${ }^{7}$ We also searched for neutrino emission from 153 additional non-triggered bursts discovered in the BATSE archival data; we observed zero events from these bursts as well. We do not include these results in the flux upper limits or MRF determinations.
} 
This is an unedited preprint of an article accepted for publication in The Astrophysical Journal. The final published article may differ from this preprint. Copyright 2007 by The American Astronomical Society. Please cite as 'ApJ preprint doi:10.1086/'524920".

Furthermore, the flux upper limit determined for this model is derived from observations of long bursts only. As with the results of Murase \& Nagataki (2006a), if this model is expanded to include short-duration bursts, the flux upper limit improves by approximately $13 \%$. However, only a very small number of all bursts $(\sim 4$ out of many thousands) have been observed in association with SNe. And, as described in Section 2, at least a fraction of these SNe did not occur at an ideal time relative to the burst. Thus, AMANDA's results confirm previous observations that lead us to expect less than maximal emission from this model of GRB neutrino production.

Finally, we observe zero events (on an expected background of 0.2 events) from the precursor time period of the bursts from 2001-2003 (Table 6). The precursor model of neutrino production was tested for only a small subset of the long-duration bursts, and the neutrino energy spectrum peaks at a level where the AMANDA-II sensitivity is greatly reduced. Thus, the flux upper limit for the precursor model is significantly less restrictive.

The results of these analyses can also be applied to any other hypothesized spectrum by using the Green's Function Fluence Limit formula, in a method similar to that presented by the Super-Kamiokande Collaboration (Fukuda et al. 2002). By folding the energy-dependent sensitivity of the detector into a desired theoretical spectrum, one can straightforwardly calculate a flux upper limit for that specific spectrum. The Green's Function fluence upper limit for AMANDA-II (Figure 10) extends several orders of magnitude in energy beyond the range of the Super-Kamiokande limit, and is approximately an order of magnitude lower than the Super-Kamiokande results in the region of overlap, primarily due to the much larger effective area of AMANDA-II. For example, at $100 \mathrm{TeV}$ we calculate $F_{\nu} \leq 1.7 \times 10^{-7}$ $\mathrm{cm}^{-2}$ (see also Appendix B). As this method does not rely on averaging burst properties (as many specific models do), it is particularly effective for incorporating large burst-to-burst variations in expected muon neutrino flux (e.g. for GRB030329, see Stamatikos 2006).

\section{Conclusion and Outlook}

The AMANDA dataset has been searched for muon neutrino emission from more than 400 GRBs based on temporal and spatial coincidence with photon detections from numerous other observatories. We determined that the detector was operating in a stable fashion during all of these bursts, and we have shown that the application of a number of data selection criteria lead to an optimized value of the Model Rejection Factor for the WaxmanBahcall neutrino spectrum. After the application of these criteria, zero neutrino events were observed in coincidence with the bursts, resulting in the most stringent upper limit on the muon neutrino flux from GRBs to date. We have compared this limit to the flux predictions 
This is an unedited preprint of an article accepted for publication in The Astrophysical Journal. The final published article may differ from this preprint. Copyright 2007 by The American Astronomical Society. Please cite as 'ApJ preprint doi:10.1086/'524920".

from several prominent GRB models based on averaged burst properties. We constrain the parameter space of a number of these models at the $90 \%$ confidence level; in particular, our flux upper limit is more than a factor of 2 below the most optimistic predictions of Razzaque et al. However, we do not yet rule out the predictions of the canonical Waxman \& Bahcall model. Likewise, our null result means that we cannot exclude the possibility that GRBs are powered by the Poynting flux mechanism rather than by the fireball mechanism. Furthermore, because individual bursts vary significantly in their expected neutrino spectra, we have presented a spectrum-independent method for determining flux upper limits for these bursts. The observations detailed in this work will play a significant role as future analyses seek to further constrain various theoretical models.

Finally, AMANDA's search for muon neutrinos from more recent GRBs will benefit greatly from the advanced capabilities of the Swift satellite (Burrows et al. 2005), as will the GRB searches of other neutrino observatories currently in operation (Spiering et al. 2004; Resvanis et al. 2003; Aguilar et al. 2006). While Swift's rate of GRB detections is lower than that of BATSE, the spatial localizations of the bursts by Swift are much more precise, which will obviate the need for a special analysis of poorly-localized bursts with its accompanying reduction in signal detection efficiency. Additionally, the InterPlanetary Network of satellites will continue to operate, and will incorporate newer instruments as they come online. In particular, future missions such as the Gamma-Ray Large Area Space Telescope (Carson 2006) will provide an even greater number of GRB localizations for use in neutrino searches. Furthermore, while analyses similar to the one presented here will continue to search specifically for muon neutrino flux in coincidence with photon observations of gammaray bursts, the method described here can be expanded to search for neutrinos correlated with other transient point sources as well (see Appendix C). In the future, AMANDA and its successor, IceCube, will have many more opportunities to detect neutrino emission from a host of astrophysical sources. Construction of IceCube is currently underway, and the instrumented volume for the partial detector is already significantly larger than the final instrumented volume of AMANDA. A fully-instrumented IceCube detector will be 20-30 times more sensitive than AMANDA, and should surpass AMANDA's flux upper limits within its first few years of operation.

The authors are thankful to E. Waxman and K. Murase for productive and thoughtprovoking discussions, S. Desai for detailed discussions regarding the Super-Kamiokande GRB analysis, and the additional members of the Interplanetary Network for providing data that were crucial to this work. The IceCube collaboration acknowledge the support from the following agencies: National Science Foundation-Office of Polar Program, National Science Foundation-Physics Division, University of Wisconsin Alumni Research Foundation, 
This is an unedited preprint of an article accepted for publication in The Astrophysical Journal. The final published article may differ from this preprint. Copyright 2007 by The American Astronomical Society. Please cite as 'ApJ preprint doi:10.1086/'524920".

Department of Energy, and National Energy Research Scientific Computing Center (supported by the Office of Energy Research of the Department of Energy), the NSF-supported TeraGrid system at the San Diego Supercomputer Center (SDSC), and the National Center for Supercomputing Applications (NCSA); Swedish Research Council, Swedish Polar Research Secretariat, and Knut and Alice Wallenberg Foundation, Sweden; German Ministry for Education and Research, Deutsche Forschungsgemeinschaft (DFG), Germany; Fund for Scientific Research (FNRS-FWO), Flanders Institute to encourage scientific and technological research in industry (IWT), Belgian Federal Office for Scientific, Technical and Cultural affairs (OSTC); the Netherlands Organisation for Scientific Research (NWO); M. Ribordy acknowledges the support of the SNF (Switzerland); A. Kappes and J. D. Zornoza acknowledge the Marie Curie OIF Program (contract 007921). K. Hurley is grateful for IPN support under the following contractsand grants: Ulysses, JPL958056; Mars Odyssey, JPL 1282043; HETE, MIT-SC-R-293291; the U.S. BeppoSAX Guest Investigator program; the U.S. INTEGRAL Guest Investigator program. Integration of NEAR into the Interplanetary Network was supported by NASA's NEAR Participating Scientist program. Integration of RHESSI was supported by NASA's Long Term Space Astrophysics program under grant NAG5-13080.

\section{A. Model-Dependent Statistical Corrections to Flux Upper Limits}

Though the $\nu$ flux formulation of Waxman (2003) explicitly links GRB neutrinos to the UHECR flux, elsewhere a formulation based on BATSE observations is treated in a comparable fashion, and is considered to arise from the same underlying phenomena (Waxman \& Bahcall 1997). Thus it is necessary to address the limitations introduced by AMANDA's reliance upon BATSE observations. As described in Section 3.4, models defined initially in terms of BATSE observations were also applied to bursts detected by the other IPN satellites. However, we cannot assume that characteristics of bursts detected by satellites with different sensitivities are completely identical. Since BATSE was decommissioned in May of 2000, there is no longer a way to cross-correlate the two datasets. Non-triggered BATSE bursts have on average less than 1/10 of the peak photon flux of the triggered bursts; assuming that the energy of neutrinos scales with the energy carried by gamma rays, we expect only a small fraction of the standard neutrino flux from these non-triggered bursts. Thus, if non-triggered bursts are inadvertently included in the flux upper limit, they will artificially improve that limit, because the extra bursts are assumed to have a larger neutrino flux than they would actually possess.

During the period of simultaneous operation from 1991 to 2000, 1088 IPN bursts were observed by BATSE, 953 of which were triggered. Undoubtedly some of these bursts did not 
This is an unedited preprint of an article accepted for publication in The Astrophysical Journal. The final published article may differ from this preprint. Copyright 2007 by The American Astronomical Society. Please cite as 'ApJ preprint doi:10.1086/'524920".

trigger BATSE for reasons other than a lower flux. For example, BATSE may have been powered down, may have been in the vicinity of the South Atlantic Anomaly, or may have experienced unrelated on-board performance problems. However, we assume conservatively that all such bursts did in fact exhibit the lower flux common to non-triggered bursts. Therefore, $\sim 12 \%$ of the IPN bursts should not actually be a part of the dataset that is compared with the models that are based upon BATSE's triggered GRB rate. Because IPN bursts are expected to contribute $\sim 25 \%$ of our detectable signal, this effect reduces the total expected neutrino flux by $\sim 3 \%$. This correction is applied asymmetrically to the overall uncertainty, because it can hinder, but not improve, the effectiveness of the analysis (see Table 4).

For models based solely on long-duration bursts, such as (Murase \& Nagataki 2006a; Razzaque et al. 2003a), the inclusion of bursts of unknown duration may also lead to an overestimation of the expected signal events, and thus a flux upper limit that is too restrictive. In order to ensure that we would not exclude potentially detectable neutrino events, the 75 bursts of unknown duration included in the dataset are assumed to last $100 \mathrm{~s}$ (for 19971999) or $50 \mathrm{~s}$ (for 2000-2003). Thus, for purposes of data analysis, they are classified as long-duration bursts. However, this necessitates a statistical correction to the resulting flux limits. We assume that up to $1 / 3$ of these bursts may in fact be short-duration, based upon the standard ratio of short- to long-duration bursts observed by BATSE. So, of the 389 bursts known (or assumed) to be long-duration, 25 were excluded from the relevant limits, thus reducing the expected number of signal events by $25 / 389$, or $\sim 6 \%$. This correction is likewise applied asymmetrically to the overall uncertainty.

\section{B. Green's Function Fluence Upper Limit Calculation}

We show here sample calculations of the differential neutrino fluence upper limit, as well as a procedure to determine the integrated fluence and flux upper limits, following the Green's Function method set out in Section 3 of Fukuda et al. (2002). The fluence upper limit calculation assumes a monochromatic neutrino spectrum; the calculation is repeated at different values of the neutrino energy. The benefit of this method is that an integrated fluence upper limit can then be determined for any input spectrum, whether it be based on all of the bursts in this dataset or only on a subset of all bursts.

The fluence upper limit is defined as

$$
F(E) \leq \frac{\mathrm{N}_{90}}{\mathrm{~A}_{\mathrm{eff}, \nu}\left(\mathrm{E}_{\nu}\right)}
$$

where $\mathrm{N}_{90}$ is $\mu_{90} / \mathrm{N}_{\text {Bursts }}$ and $\mathrm{A}_{\mathrm{eff}, \nu}$ is the energy-dependent neutrino effective collecting area 
This is an unedited preprint of an article accepted for publication in The Astrophysical Journal. The final published article may differ from this preprint. Copyright 2007 by The American Astronomical Society. Please cite as 'ApJ preprint doi:10.1086/'524920".

(see Section 3.3 $)^{8}$.

Figure 10 is determined by the results of AMANDA's 2000-2003 observations. For example, $\mu_{90}=1.30$ and $\mathrm{N}_{\text {Bursts }}=151$; therefore, $\mathrm{N}_{90}=8.61 \times 10^{-3}$.

For $E_{\nu}=100 \mathrm{TeV}$ (near the peak of the predicted neutrino flux), $A_{\text {eff }, \nu}=5.0 \times 10^{4}$ $\mathrm{cm}^{2}$ therefore $\mathrm{F}(100 \mathrm{TeV}) \leq 1.7 \times 10^{-7} \mathrm{~cm}^{-2}$.

We now determine the integrated fluence upper limit explicitly for an $\mathrm{E}^{-2}$ spectrum, as well the Waxman-Bahcall spectrum. First, the integrated fluence, $\mathrm{F}_{\text {int }}$ for an $\mathrm{E}^{-2}$ spectrum is

$$
F_{\text {int }} \leq\left[\int_{250 \mathrm{GeV}}^{10^{7} \mathrm{GeV}} \frac{C E_{\nu}^{-2}}{F(E)} \mathrm{dE}_{\nu}\right]^{-1}=1.4 \times 10^{-5} \mathrm{~cm}^{-2},
$$

where $\mathrm{C}$ is the factor required to normalize the neutrino spectrum to unity - in this case, $\mathrm{C}=250 \mathrm{GeV}$. This integrated fluence upper limit is significantly lower than the results of similar calculations performed by Fukuda et al. (2002) (we combine the $\nu_{\mu}$ and $\bar{\nu}_{\mu}$ fluences into a single limit, while they present two separate fluence upper limits). However, a direct, quantitative comparison between these two results cannot be made due to the vastly different energy ranges of the two instruments. Note also the limits of integration employed herethough AMANDA is sensitive to neutrinos at higher and lower energies, the vast majority of the flux from GRBs is expected to come from neutrinos of a few hundred GeV to a few $\mathrm{PeV}$.

Now we determine the integrated fluence upper limit for the Waxman-Bahcall spectrum, to provide a further example of the wide applicability of the Green's Function method:

$$
F_{\text {int }} \leq\left[\int_{250 \mathrm{GeV}}^{10^{5} \mathrm{GeV}} \frac{C E_{\nu}^{-1} E_{\mathrm{Break}}^{-1}}{F(E)} \mathrm{dE}_{\nu}+\int_{10^{5}}^{10^{7}} \frac{C E_{\nu}^{-2}}{F(E)} \mathrm{dE}_{\nu}\right]^{-1}=5.3 \times 10^{-7} \mathrm{~cm}^{-2},
$$

where $\mathrm{C}$ again is the constant required to normalize the overall spectrum to unity; here $\mathrm{C}$ $=7.0 \times 10^{-5} \mathrm{GeV}$.

Finally, we compare this fluence upper limit to the flux upper limit derived for the Waxman-Bahcall spectrum in Section 4. To do this, we must convert the integrated fluence upper limit into a differential all-sky flux upper limit per burst; that is, from units of $\mathrm{cm}^{-2}$ to units of $\mathrm{GeV}^{-1} \mathrm{~cm}^{-2} \mathrm{~s}^{-1} \mathrm{sr}^{-1}$ :

\footnotetext{
${ }^{8}$ Instead of using the neutrino effective area, one could also use the muon effective area multiplied by the neutrino to muon conversion probability (as in Fukuda et al. 2002); in the case of AMANDA one must also account for attenuation of neutrinos in the earth.
} 
This is an unedited preprint of an article accepted for publication in The Astrophysical Journal. The final published article may differ from this preprint. Copyright 2007 by The American Astronomical Society. Please cite as 'ApJ preprint doi:10.1086/'524920".

$$
\frac{F_{\text {int }}}{\Omega \mathrm{t}}=\frac{5.3 \times 10^{-7}}{(4 \pi)\left(3.15 \times 10^{7} / 700\right)}=9.4 \times 10^{-13} \mathrm{~cm}^{-2} \mathrm{~s}^{-1} \mathrm{sr}^{-1}
$$

Next, we multiply by the normalization of the energy spectrum and take the differential to provide a flux upper limit of

$\mathrm{E}^{2} \Phi_{\nu} \leq 1.3 \times 10^{-8} \mathrm{GeVcm}^{-2} \mathrm{~s}^{-1} \mathrm{sr}^{-1}$.

This is nearly identical to the flux upper limit derived in the manner described in Section 3 for 151 bursts from 2000 to 2003 (see also Table 5, where an MRF of 2.6 yields a flux upper limit of $\mathrm{E}^{2} \Phi_{\nu} \leq 1.2 \times 10^{-8} \mathrm{GeVcm}^{-2} \mathrm{~s}^{-1} \mathrm{sr}^{-1}$, consistent with the result derived above to within the applicable uncertainties).

Thus we show that the Green's Function Method agrees with calculations which explicitly incorporated prior assumptions about the GRB neutrino spectrum. Therefore, this alternate method provides a powerful tool for determining the flux upper limit based on AMANDA observations for any proposed neutrino spectrum.

\section{Expanding the GRB Search to Other Transient Point Sources}

While this work has provided the most stringent upper limit to date specifically for muon neutrino flux for gamma-ray bursts in coincidence with photon observations, the method described above can be expanded to search for other transient point sources as well. X-ray flares occurring minutes to hours after a GRB are thought to be caused by re-activation of the GRB central engine, and are a natural candidate for correlated neutrino searches (Murase \& Nagataki 2006b). Additionally, photon emission from supernovae could be used as a key element in searches for neutrino emission from jet-driven supernovae and $\gamma$-ray dark ("choked") GRBs (Razzaque et al. 2003b). Jet-driven supernovae are expected to accelerate baryonic material to mildly relativistic energies (the Lorentz boost $\Gamma \sim$ a few), which may subsequently result in significant neutrino emission (Ando \& Beacom 2005). Not all supernovae will be jet-driven, but population estimates vary between $0.2 \%$ and $25 \%$ of all type Ib/c SNe (van Putten 2004; Berger et al. 2003; Soderberg 2005). Given the number of such supernovae observed annually, it is reasonable to search for a neutrino signal from these events.

Another reason to search for neutrino emission from supernovae becomes apparent when

we consider the recently-established SN-GRB connection. Several supernovae (including 1998bw and 2003dh) are known to be associated with GRBs. Furthermore, Razzaque et al. (2003b) describe a scenario where as many as $10^{3}$ times the standard number of GRBs occur, 
This is an unedited preprint of an article accepted for publication in The Astrophysical Journal. The final published article may differ from this preprint. Copyright 2007 by The American Astronomical Society. Please cite as 'ApJ preprint doi:10.1086/'524920".

though in these bursts the photon jet does not succeed in escaping the stellar envelope (the $\gamma$-ray dark GRBs). For these types of bursts, no gamma-rays will be observed. However, if even a fraction of these GRBs are associated with SNe (the fraction for observed GRBs has been calculated to be in the range of $10^{-2}$ to $10^{-3}$ (Bissaldi et al. 2006)), then it will be possible to search for neutrinos in the time period surrounding the SN emission (provided the SN start time, the GRB time delay relative to the SN, and the duration of the GRB can be estimated with sufficient precision). Because these SNe are localized transient phenomena, the primary selection criteria for the GRB analysis (spatial and temporal correlation) are an excellent starting point for such a search, though it is possible that not all of the other data quality cuts used in the GRB search would be optimal for a supernova search. Finally, it is also possible to complement any of the transient point source searches described above by inverting the search algorithm, that is, by implementing Target of Opportunity photon searches based on spatio-temporal localization of potential neutrino events (Kowalski \& Mohr 2007). Any of these searches can potentially be of great benefit to the long-term goals of multi-messenger astronomy.

\section{REFERENCES}

Achterberg, A. et al., ApJ, in press (astro-ph/0702265)

Achterberg, A. et al., submitted to PRD, November 2006 (astro-ph/0611063)

Achterberg, A. et al., Astropart. Phys. 26 (2006) 155 (astro-ph/0604450)

Ackermann, M. et al., J. Geophys. Res. 111 (2006), D13203

Aguilar, A.J. et al., Astropart. Phys. 26 (2006) 314-324; see also the Antares Neutrino Telescope Page: http://antares.in2p3.fr

Aharmim, B. et al., NIM A449 (2000) 172-207

Ahrens, J. et al., NIM A54 (2004) 169

Ahrens, J. et al., PRL 90 (2003) 251101

Ahrens, J. et al., ApJ 583 (2003) 1040

Ahrens, J. et al., PRD 66 (2002) 012005

Andrés, E. et al., Nature 410 (2001) 441-443

Ando, S. and J. Beacom, PRL 95 (2005) 061103 
This is an unedited preprint of an article accepted for publication in The Astrophysical Journal. The final published article may differ from this preprint. Copyright 2007 by The American Astronomical Society. Please cite as 'ApJ preprint doi:10.1086/'524920".

Bay, R.C. Ph.D. Thesis, University of California (2000) (astro-ph/0008255)

Berger, E. et al., ApJ 599 (2003) 408

Bissaldi, E. et al., A\&A, in press (astro-ph/0702652)

Burrows, D. et al., Space Sci. Rev. 120 (2005) 165; see also the Swift Gamma-Ray Burst Mission Page: http://swift.gsfc.nasa.gov/docs/swift/swiftsc.html

Carson, J. for the GLAST Collaboration, Proc. 2nd TeV Workshop, Madison, WI (2006) (astro-ph/0610960); see also the GLAST Mission Page: http://glast.gsfc.nasa.gov

Chirkin, D. and W. Rhode, hep-ph/0407075

Conrad, J. et al., PRD 67 (2003) 012002

Costa, E. et al., Nature 386 (1997) 686-688

Dermer, C. and A. Atoyan, New Journal of Physics 8 (2006) 122 (astro-ph/0606629)

Dermer, C. and A. Atoyan, PRL 91 (2003) 1102

Eicher, D. et al., Nature 340 (1989) 126-128

Feldman, G. and R. Cousins, PRD 57 (1998) 3873-3889

Fishman, G.J. et al., A\&A Supp 97 (1993) 17-20

Frail, D. et al., ApJ 562 L55-58 (2001)

Fukuda, S. et al., ApJ 578 (2002) 317

Gandhi, R. et al., PRD 58 (1998) 093009

Gandhi, R. et al., Astropart. Phys. 5 (1996) 81-110

Goodman, A., ApJ 308 L47 (1986)

Hardtke, R., Ph.D Thesis, University of Wisconsin (2002)

Heck, D. et al., Report FZKA 6019 (1998). An update of the original Corsika (6.030) was used in this analysis. See http://www-ik.fzk.de/corsika/ for additional information and more recent versions.

Hill, G.C. and K. Rawlins, Astropart. Phys. 19 (2003) 393 
This is an unedited preprint of an article accepted for publication in The Astrophysical Journal. The final published article may differ from this preprint. Copyright 2007 by The American Astronomical Society. Please cite as 'ApJ preprint doi:10.1086/'524920".

Hill, G.C., Astropart. Phys. 6 (1997) 215

Hodges, J. for the IceCube Collaboration, Proc. 2nd TeV Workshop, Madison, WI (2006) (astro-ph/0611597)

Hurley, K. et al., in "Compact Stellar X-Ray Sources", Lewin, W. et al., Eds. Cambridge University Press, Cambridge (2006)

Hurley, K., Astron. Telegram \#19 (1998)

Kalmykov, N. and S. Ostapchenko, Phys. Atom. Nucl. 56 (1993) 346

Kashti, T. and E. Waxman, PRL 95 (2005) 181101

Kommers, J. Ph.D. Thesis, MIT (1998); see also http://space.mit.edu/BATSE

Kowalski, M. and A. Mohr, astro-ph/0701618

Kuehn, K., http://icecube.wisc.edu/ kuehn/grb9703.html (AMANDA-GRB Supplemental Information Page)

Lipari, P. and T. Stanev, PRD 44 (1991) 113543

Lyutikov, M. and R. Blandford, arXiv:astro-ph/0312347

Markwardt, C.B. et al., ApJ 633 L77-80 (2005)

Murase, K. and S. Nagataki, PRD 73 (2006) 063002

Murase, K. and S. Nagataki, PRL 97 (2006) 051101 (astro-ph/0604437)

Murase, K. and S. Nagataki, private communication

Narayan, R., B. Paczyński, and T. Piran, ApJ 395 L83 (1998)

Paczyński, B., AIP Conf. Proc. 428 (1998) 783

Paczyński, B., ApJ 494 L45 (1998)

Paczyński, B., ApJ 308 L43 (1986)

Razzaque, S. et al., PRD 68 (2003) 3001

Razzaque, S. et al., PRL 90 (2003) 241103

Rees, M. and P. Mészáros, MNRAS 258 (1992) 41-43 
This is an unedited preprint of an article accepted for publication in The Astrophysical Journal. The final published article may differ from this preprint. Copyright 2007 by The American Astronomical Society. Please cite as 'ApJ preprint doi:10.1086/'524920".

Resvanis, L.K. et al., Nuc. Phys. B Proc. Supp. 122 (2003) 24-39; see also the NESTOR Institute for Astroparticle Physics Page: http://www.nestor.org.gr

Roming, P.W.A. et al., ApJ 651 (2006) 985 (astro-ph/0605005)

Soderberg, A. Nuovo Cim. 28C (2005) 563-573

Spiering, C. et al., astro-ph/0404096

Stamatikos, M. for the IceCube Collaboration, et al. Proc. 29th ICRC, Pune, India (2005) 471-474 (astro-ph/0510336)

Stamatikos, M. for the IceCube Collaboration, and D. Band, AIP Conf. Proc. 836 (2006) 599 (astro-ph/0604281)

Stern, B.E. et al., ApJ 563 (2001) 80

van Putten, M., ApJ 611 L81-84 (2004)

Waxman, E., Nuc. Phys. B Proc. Supp. 118 (2003) 353-362 (and references therein)

Waxman, E. and J. Bahcall, PRL 78 (1997) 2292

Woosley, S., ApJ 405 (1993) 273 
This is an unedited preprint of an article accepted for publication in The Astrophysical Journal. The final published article may differ from this preprint. Copyright 2007 by The American Astronomical Society. Please cite as 'ApJ preprint doi:10.1086/'524920".

Table 1: Primary Instruments in the Third Interplanetary Network, 1997-2003

\begin{tabular}{ccc}
\hline Instrument & Energy Range (keV) & Mission Homepage \\
\hline BATSE LAD & $30-190$ & http://www.batse.msfc.nasa.gov \\
BeppoSAX GRBM & $40-700$ & http://www.asdc.asi.it/bepposax \\
BeppoSAX WFC & $2-26$ & http://www.asdc.asi.it/bepposax \\
HETE-II FREGATE & $6-400$ & http://space.mit.edu/HETE/fregate.html \\
HETE-II WXM & $2-25$ & http://space.mit.edu/HETE/wxm.html \\
HETE-II SXC & $2-14$ & http://space.mit.edu/HETE/sxc.html \\
INTEGRAL & $15-10000$ & http://integral.esac.int/ \\
Konus WIND & $12-10000$ & http://www-spof.gsfc.nasa.gov/istp/wind/ \\
Mars Odyssey & $\sim 100-8000$ & http://mars.jpl.nasa.gov/odyssey/ \\
NEAR XGRS & $100-1000$ & http://near.jhuapl.edu \\
RHESSI & $\sim 25-\sim 25000$ & http://hesperia.gsfc.nasa.gov/hessi \\
Ulysses & $25-150$ & http://ulysses.jpl.nasa.gov \\
\hline
\end{tabular}


This is an unedited preprint of an article accepted for publication in The Astrophysical Journal. The final published article may differ from this preprint. Copyright 2007 by The American Astronomical Society. Please cite as 'ApJ preprint doi:10.1086/'524920".

Table 2: BATSE Triggered and IPN Bursts Per Year in the AMANDA Analysis, by Duration

\begin{tabular}{cccccccc}
\hline Year & 1997 & 1998 & 1999 & 2000 & 2001 & 2002 & 2003 \\
\hline $\mathrm{N}_{\text {Short }}$ & 12 & 15 & 9 & 7 & 1 & 1 & 2 \\
$\mathrm{~N}_{\text {Long }}$ & 51 & 50 & 61 & 77 & 15 & 21 & 24 \\
$\mathrm{~N}_{\text {Unknown }}$ & 15 & 29 & 26 & 3 & 0 & 0 & 0 \\
\hline $\mathrm{N}_{\text {Total }}$ & 78 & 94 & 96 & 87 & 16 & 22 & 26 \\
\hline
\end{tabular}


This is an unedited preprint of an article accepted for publication in The Astrophysical Journal. The final published article may differ from this preprint. Copyright 2007 by The American Astronomical Society. Please cite as 'ApJ preprint doi:10.1086/'524920".

Table 3: Data Selection Criteria, Year by Year

\begin{tabular}{ccccc}
\hline Criterion & $97-99$ & 00 & $01-03$ & Precursor $^{\mathrm{a}}$ \\
\hline$\Delta \Psi_{1}, \delta \geq 10^{\circ}\left(\delta<10^{\circ}\right)$ & $<20^{\circ}\left(<6.5^{\circ}\right)$ & $<12.5^{\circ}\left(<7^{\circ}\right)$ & $<12^{\circ}\left(<8^{\circ}\right)$ & $<12^{\circ}\left(<5^{\circ}\right)$ \\
$\Delta \Psi_{2}, \delta \geq 10^{\circ}\left(\delta<10^{\circ}\right)$ & $\mathrm{N} / \mathrm{A}$ & $\mathrm{N} / \mathrm{A}$ & $<12^{\circ}\left(<8^{\circ}\right)$ & $<12^{\circ}\left(<6^{\circ}\right)$ \\
$\Delta \Psi_{3}, \delta \geq 10^{\circ}\left(\delta<10^{\circ}\right)$ & $\mathrm{N} / \mathrm{A}$ & $\mathrm{N} / \mathrm{A}$ & $<16^{\circ}\left(<8^{\circ}\right)$ & $<16^{\circ}\left(<8^{\circ}\right)$ \\
$\Delta \Psi_{4}, \delta \geq 10^{\circ}\left(\delta<10^{\circ}\right)$ & $\mathrm{N} / \mathrm{A}$ & $\mathrm{N} / \mathrm{A}$ & $\mathrm{N} / \mathrm{A}$ & $<40^{\circ}\left(<40^{\circ}\right)$ \\
$\sigma_{\Psi}{ }^{\mathrm{b}}$ & $\mathrm{N} / \mathrm{A}$ & $\mathrm{N} / \mathrm{A}$ & $<5^{\circ}\left(<5^{\circ}\right)$ & $<5^{\circ}\left(<5^{\circ}\right)$ \\
Track Uniformity & $\mathrm{N} / \mathrm{A}$ & $<0.29(<0.29)$ & $<0.55(<0.55)$ & $<0.55(<0.55)$ \\
$£_{\text {reco }}{ }^{\mathrm{c}}$ & $\mathrm{N} / \mathrm{A}$ & $<7.85(<7.5)$ & $\mathrm{N} / \mathrm{A}$ & $\mathrm{N} / \mathrm{A}$ \\
Direct Hits & $>10$ & $\mathrm{~N} / \mathrm{A}$ & $\mathrm{N} / \mathrm{A}$ & $\mathrm{N} / \mathrm{A}$ \\
$N_{O M s}$ in Event & $\mathrm{N} / \mathrm{A}$ & $\mathrm{N} / \mathrm{A}(>24)$ & $\mathrm{N} / \mathrm{A}$ & $\mathrm{N} / \mathrm{A}$ \\
\hline Signal Passing Rate & $0.35(0.22)$ & $0.69(0.54)$ & $0.68(0.61)$ & 0.69 \\
\hline Expected $N_{\nu}{ }^{\mathrm{d}}$ & 0.31 & 0.28 & 0.22 & $\mathrm{~N} / \mathrm{A}$ \\
\hline
\end{tabular}

${ }^{a}$ The precursor time period was searched only during the 2001-2003 dataset.

${ }^{b}$ The angular resolution of the paraboloid fit.

${ }^{c}$ The $\log$ (Likelihood) of the reconstructed track.

${ }^{d}$ Based on the flux of Waxman (2003), corrected for neutrino oscillations. 
This is an unedited preprint of an article accepted for publication in The Astrophysical Journal. The final published article may differ from this preprint. Copyright 2007 by The American Astronomical Society. Please cite as 'ApJ preprint doi:10.1086/'524920".

Table 4: Uncertainties/Corrections in the GRB Analysis

\begin{tabular}{ccc}
\hline Source of Uncertainty & Quantity & Reference \\
\hline OM sensitivity & $\pm 7 \%$ & (Ahrens et al. 2004) \\
Simulation parameters (incl. ice properties) & $\pm 15 \%$ & Sections 3.3 \& 3.4 \\
Neutrino-nucleon cross-section & $\pm 3 \%$ & (Gandhi et al. 1998) \\
\hline Uncertainties added in quadrature & $\pm 17 \%$ & \\
\hline Correction for IPN bursts not modeled & $-3 \%$ & Appendix A \\
Correction for short bursts not modeled & $-6 \%$ & Appendix A \\
Correction for BATSE position uncertainty & $-2 \%$ & Section 3.4 \\
\hline Total & $+6 \% /-28 \%$ & \\
\hline
\end{tabular}


This is an unedited preprint of an article accepted for publication in The Astrophysical Journal. The final published article may differ from this preprint. Copyright 2007 by The American Astronomical Society. Please cite as 'ApJ preprint doi:10.1086/'524920".

Table 5: Results of GRB Analysis 1997-2003

\begin{tabular}{cccccc}
\hline Year & $1997-1999$ & 2000 & $2001-2003$ & $2000-2003$ & $1997-2003$ \\
\hline$N_{\text {Bursts }}$ & 268 & 87 & 64 & 151 & 419 \\
$N_{\text {BG,Exp }}$ & 0.46 & 1.02 & 0.27 & 1.29 & 1.74 \\
$N_{\text {Obs }}$ & 0 & 0 & 0 & 0 & 0 \\
Event Upper Limit & 1.98 & 1.50 & 2.30 & 1.30 & 1.10 \\
$M R F_{\mathrm{WB}}{ }^{\mathrm{a}}$ & 6.9 & 5.7 & 11 & 2.6 & 1.36 \\
$M R F_{\mathrm{MN}}{ }^{\mathrm{b}}$ & 5.4 & 3.4 & 6.8 & 1.5 & 0.92 \\
$M R F_{\mathrm{Razz}}$ & 2.6 & 1.6 & 3.3 & 0.75 & 0.45 \\
\hline
\end{tabular}

${ }^{a}$ Based on the flux of Waxman (2003), corrected for neutrino oscillations.

${ }^{b}$ Based on the flux of Murase \& Nagataki (2006a).

cBased on the "supranova" flux of Razzaque et al. (2003a). 
This is an unedited preprint of an article accepted for publication in The Astrophysical Journal. The final published article may differ from this preprint. Copyright 2007 by The American Astronomical Society. Please cite as 'ApJ preprint doi:10.1086/'524920".

Table 6: Results of Precursor Search 2001-2003

\begin{tabular}{ccccc}
\hline Year & $N_{\text {Bursts }}$ & $N_{\text {BG,Exp }}$ & $N_{\text {Obs }}$ & Event U.L. \\
\hline 2001 & 15 & 0.06 & 0 & 2.38 \\
2002 & 21 & 0.07 & 0 & 2.37 \\
2003 & 24 & 0.07 & 0 & 2.37 \\
\hline $2001-2003$ & 60 & 0.20 & 0 & 2.30 \\
\hline
\end{tabular}


This is an unedited preprint of an article accepted for publication in The Astrophysical Journal. The final published article may differ from this preprint. Copyright 2007 by The American Astronomical Society. Please cite as 'ApJ preprint doi:10.1086/'524920"'.

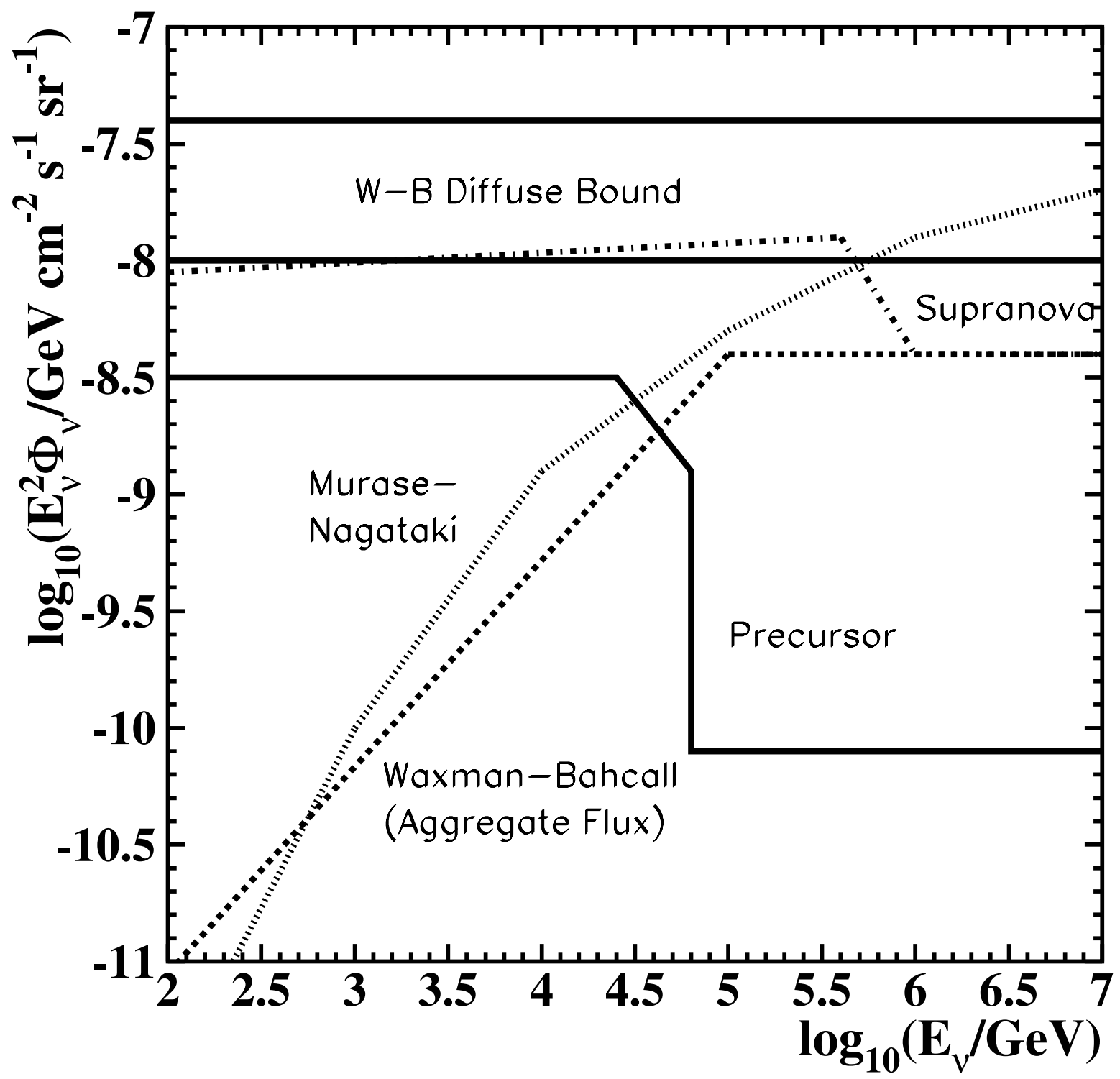

Fig. 1.- Predicted differential muon neutrino flux as a function of energy for four different models of GRB neutrino production: the precursor model (solid line), the canonical WaxmanBahcall model (thick dotted line), the Murase-Nagataki model (thin dotted line), and the supranova model (dot-dashed line). All models include the effect of $\nu$ oscillations. The diffuse neutrino bounds determined from cosmic ray observations with (upper horizontal line) and without (lower horizontal line) z evolution are also shown for reference. 
This is an unedited preprint of an article accepted for publication in The Astrophysical Journal. The final published article may differ from this preprint. Copyright 2007 by The American Astronomical Society. Please cite as 'ApJ preprint doi:10.1086/'524920".

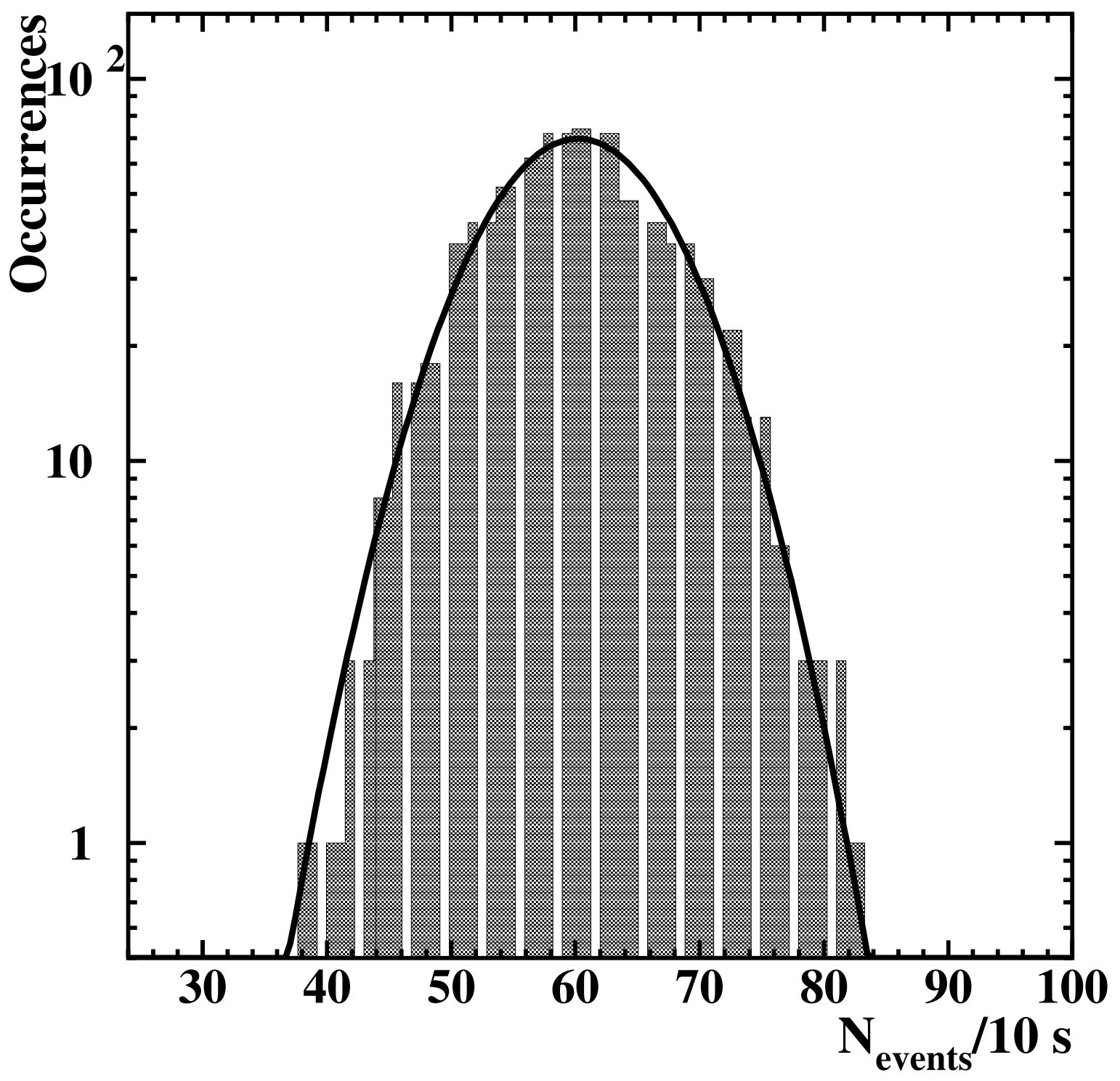

Fig. 2.- A stable period of detector activity, shown by the nearly Gaussian random temporal distribution of events in each 10-second bin during the off-time period of BATSE GRB 6610. Initial selection criteria have been applied to these data, but the GRB-specific criteria have not yet been applied. 
This is an unedited preprint of an article accepted for publication in The Astrophysical Journal. The final published article may differ from this preprint. Copyright 2007 by The American Astronomical Society. Please cite as 'ApJ preprint doi:10.1086/'524920".

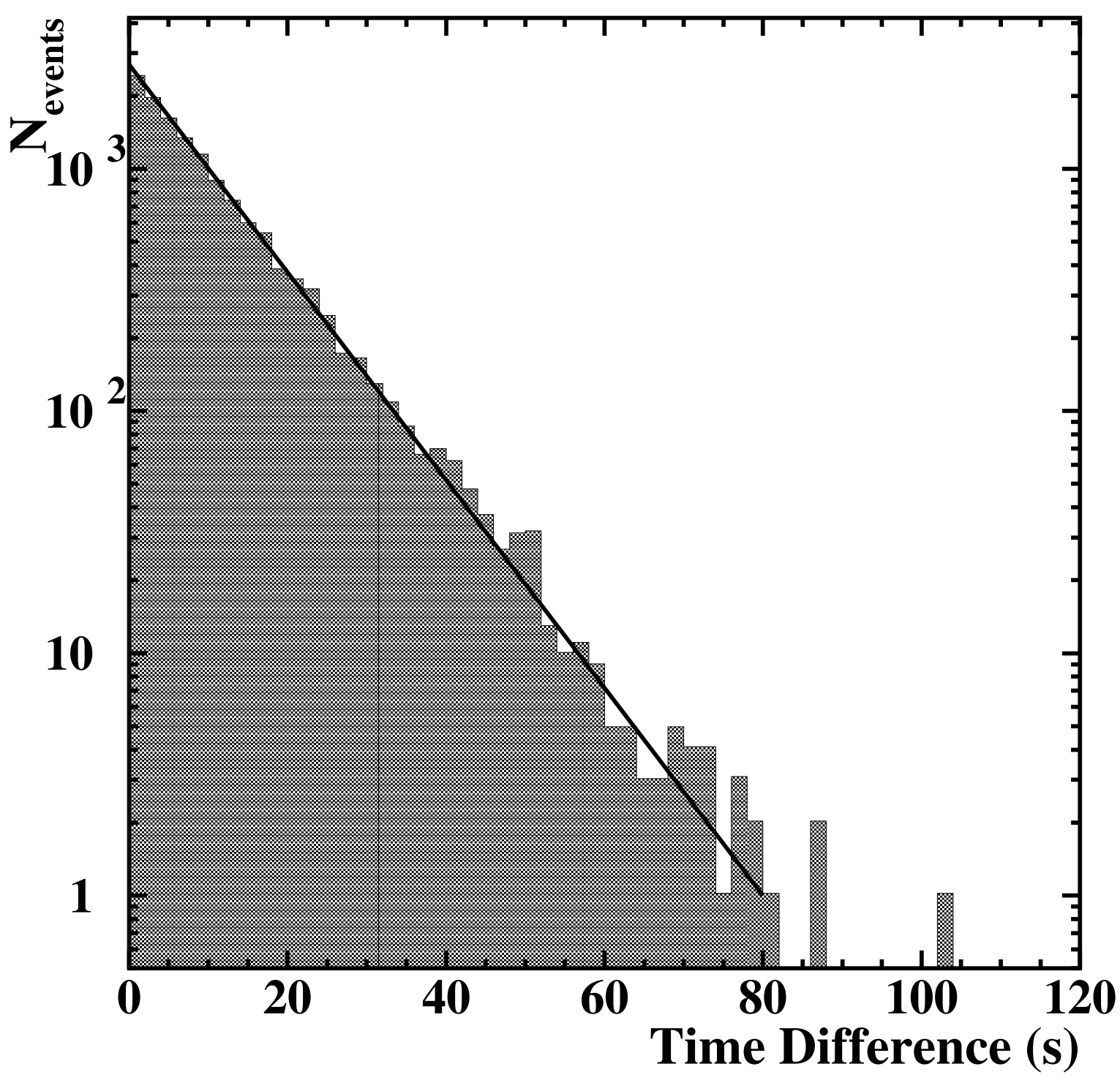

Fig. 3.- Time difference $(\delta \mathrm{t})$ between subsequent events during the background time period of a representative GRB, after application of initial data quality cuts. There is no evidence for significant gaps in the data that could produce a "false negative" result. 
This is an unedited preprint of an article accepted for publication in The Astrophysical Journal. The final published article may differ from this preprint. Copyright 2007 by The American Astronomical Society. Please cite as 'ApJ preprint doi:10.1086/'524920".

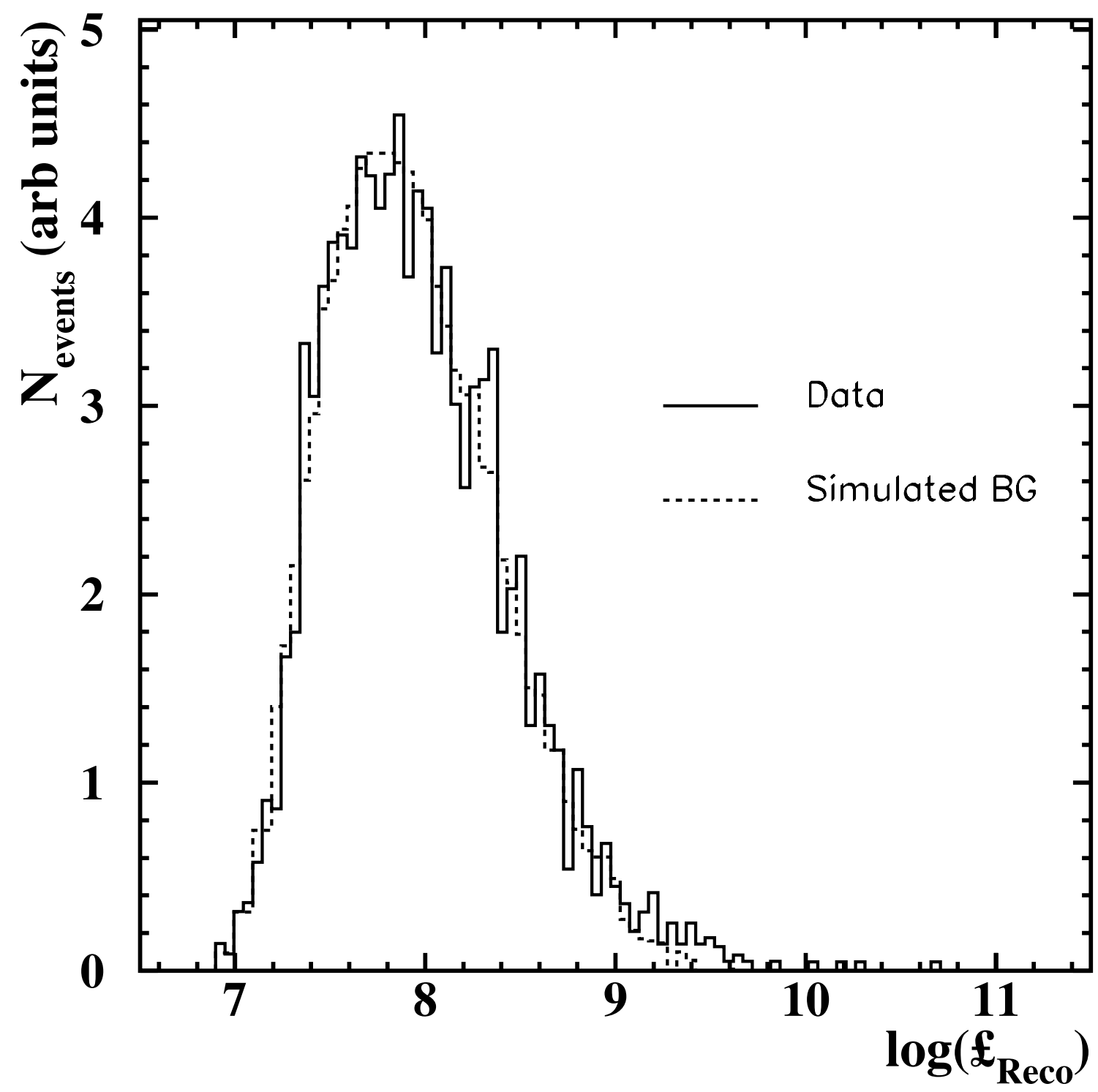

Fig. 4. - A comparison of the likelihood of track reconstruction, $£_{\text {reco }}$ for observed data (solid line) and simulated background events (dashed line). Both curves are normalized after preliminary data selection criteria are applied. The close agreement signifies that our simulations are properly modeling the observed events, thus providing additional evidence for the trustworthiness of the simulated signal events as well. 
This is an unedited preprint of an article accepted for publication in The Astrophysical Journal. The final published article may differ from this preprint. Copyright 2007 by The American Astronomical Society. Please cite as 'ApJ preprint doi:10.1086/'524920"'.

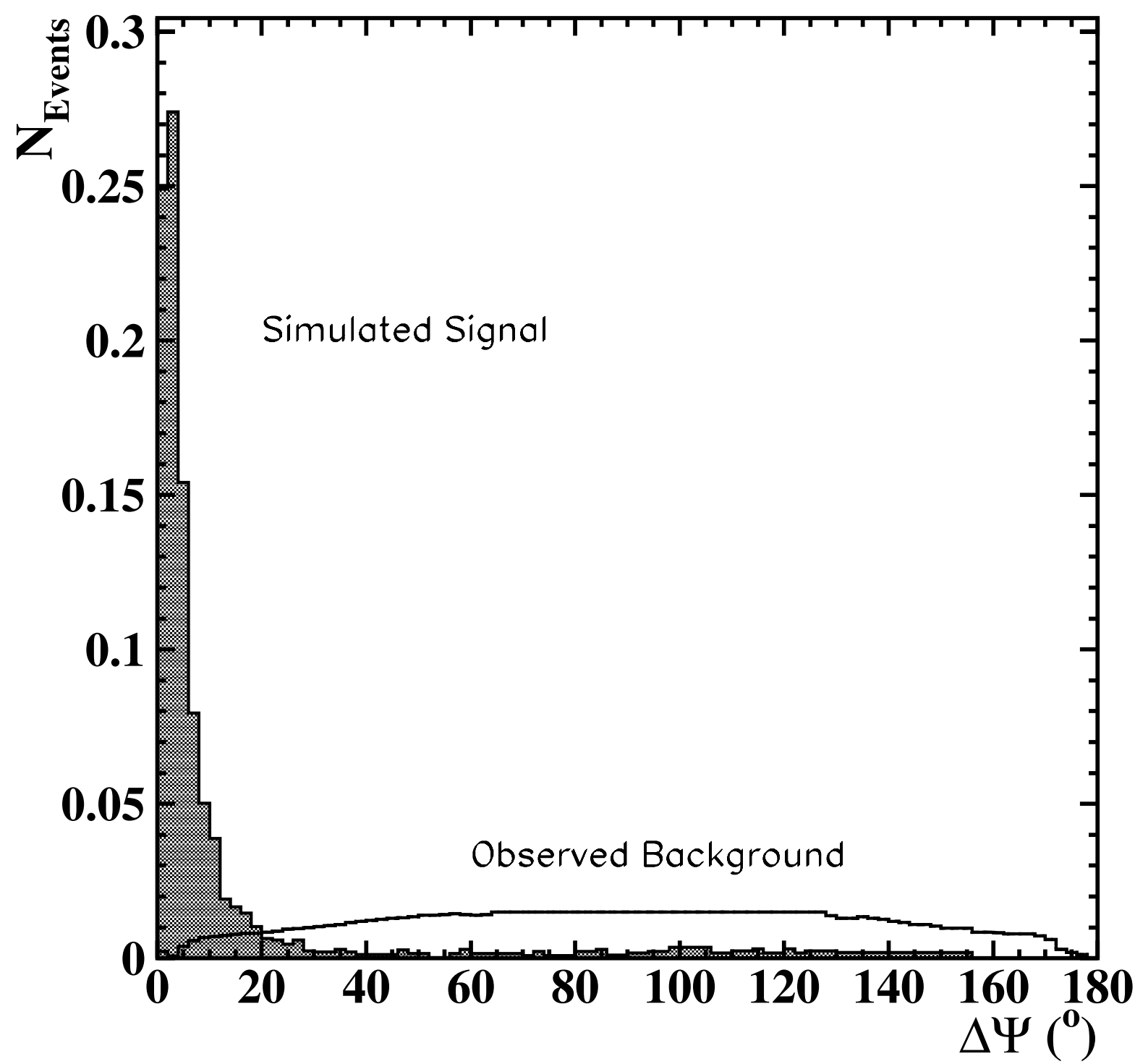

Fig. 5.- The expected distribution of angular mismatch $\Delta \Psi_{1}$ for a simulated muon neutrino spectrum (shaded region) and observed background (open region). $\Delta \Psi_{1}=0$ is the position of the burst determined from photon observations. Selecting events with $\Delta \Psi_{1} \leq 12^{\circ}$ retains more than $90 \%$ of the signal events. 
This is an unedited preprint of an article accepted for publication in The Astrophysical Journal. The final published article may differ from this preprint. Copyright 2007 by The American Astronomical Society. Please cite as 'ApJ preprint doi:10.1086/'524920"'.

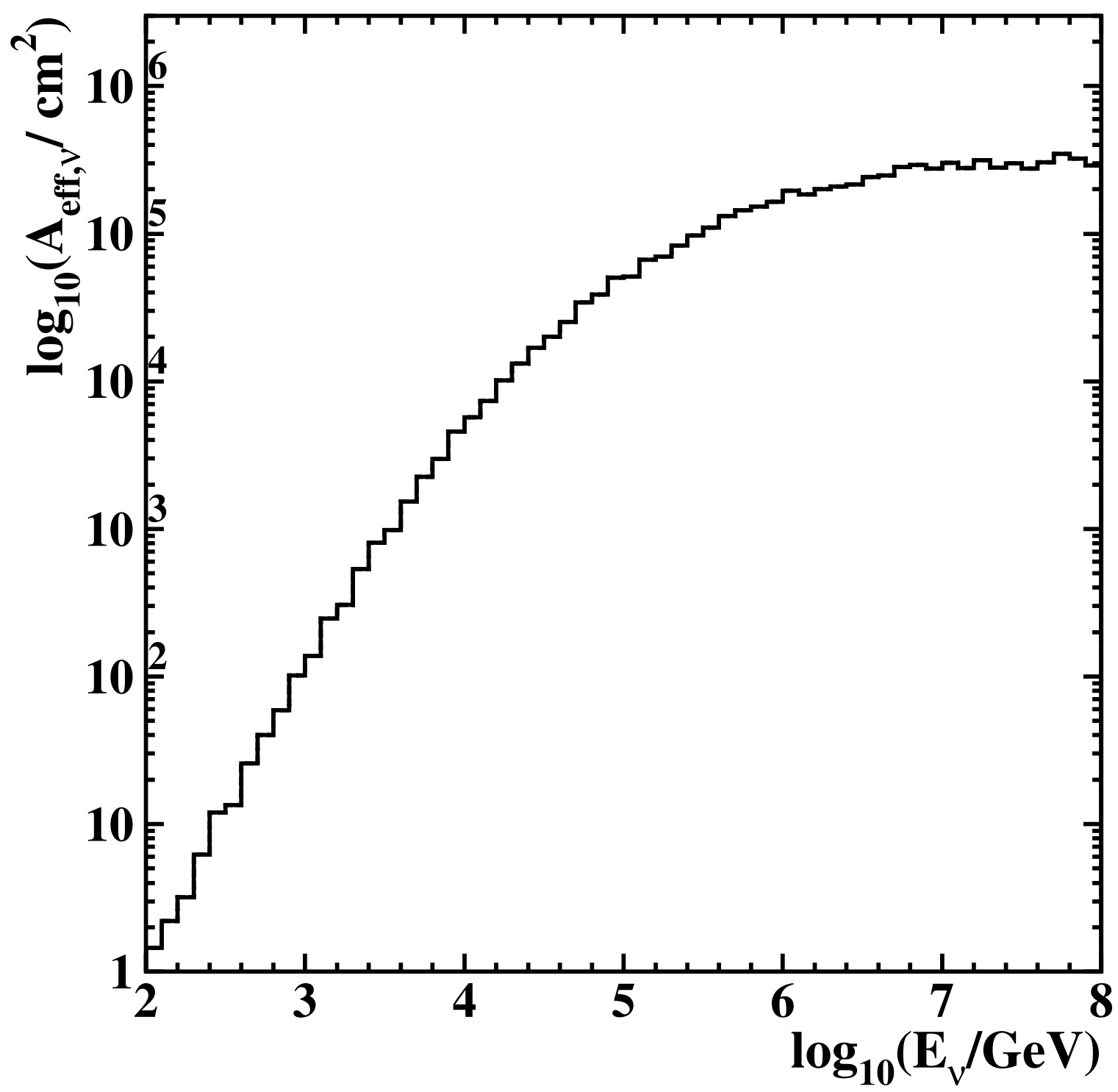

Fig. 6.- Angle-averaged muon neutrino effective area for the AMANDA-II (years 20012003) coincident search algorithm, based upon Monte Carlo simulations of expected signal events from the Northern hemisphere. 
This is an unedited preprint of an article accepted for publication in The Astrophysical Journal. The final published article may differ from this preprint. Copyright 2007 by The American Astronomical Society. Please cite as 'ApJ preprint doi:10.1086/'524920"'.

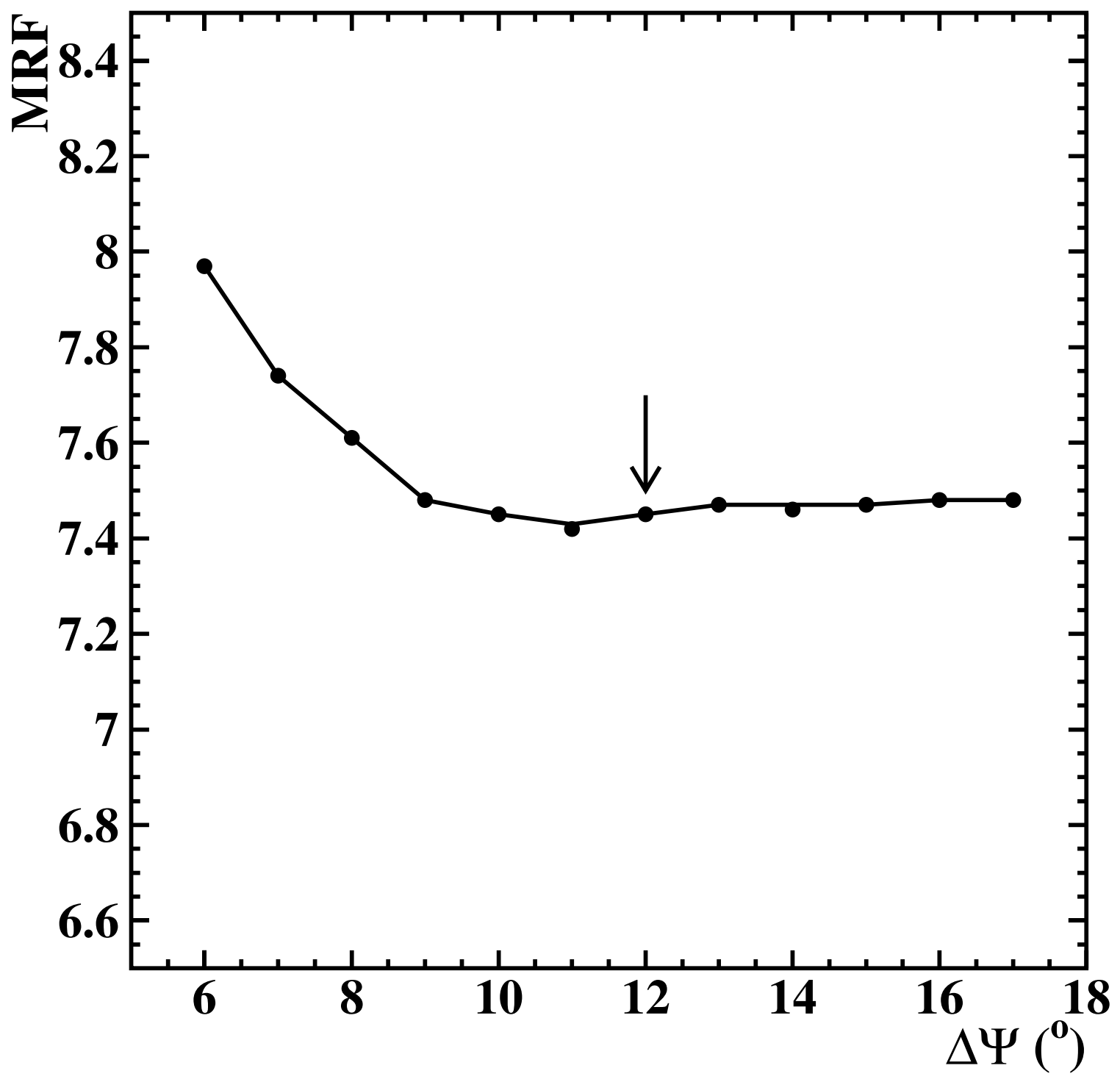

Fig. 7.- Relative Model Rejection Factor (MRF) as a function of angular mismatch $\left(\Delta \Psi_{1}\right)$ between the burst position and the reconstructed track, for the subset of bursts from 2000. The arrow indicates the mismatch angle selected for this analysis. 
This is an unedited preprint of an article accepted for publication in The Astrophysical Journal. The final published article may differ from this preprint. Copyright 2007 by The American Astronomical Society. Please cite as 'ApJ preprint doi:10.1086/'524920"'.

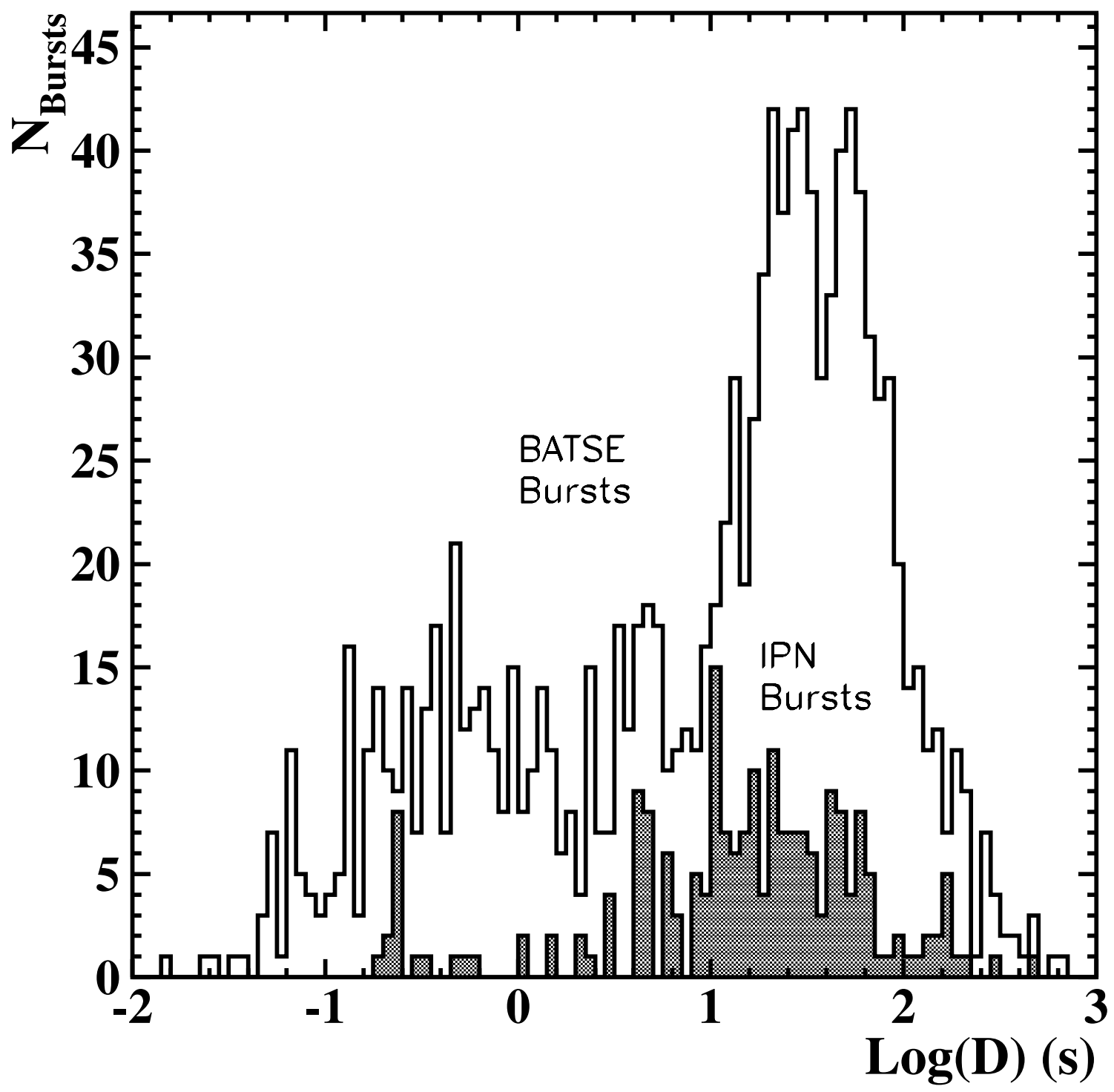

Fig. 8.- Duration distribution of BATSE GRBs (upper, open histogram) and IPN bursts for which durations have been determined (lower, shaded histogram). Both distributions appear to be drawn from the same underlying population. 
This is an unedited preprint of an article accepted for publication in The Astrophysical Journal. The final published article may differ from this preprint. Copyright 2007 by The American Astronomical Society. Please cite as 'ApJ preprint doi:10.1086/'524920"'.

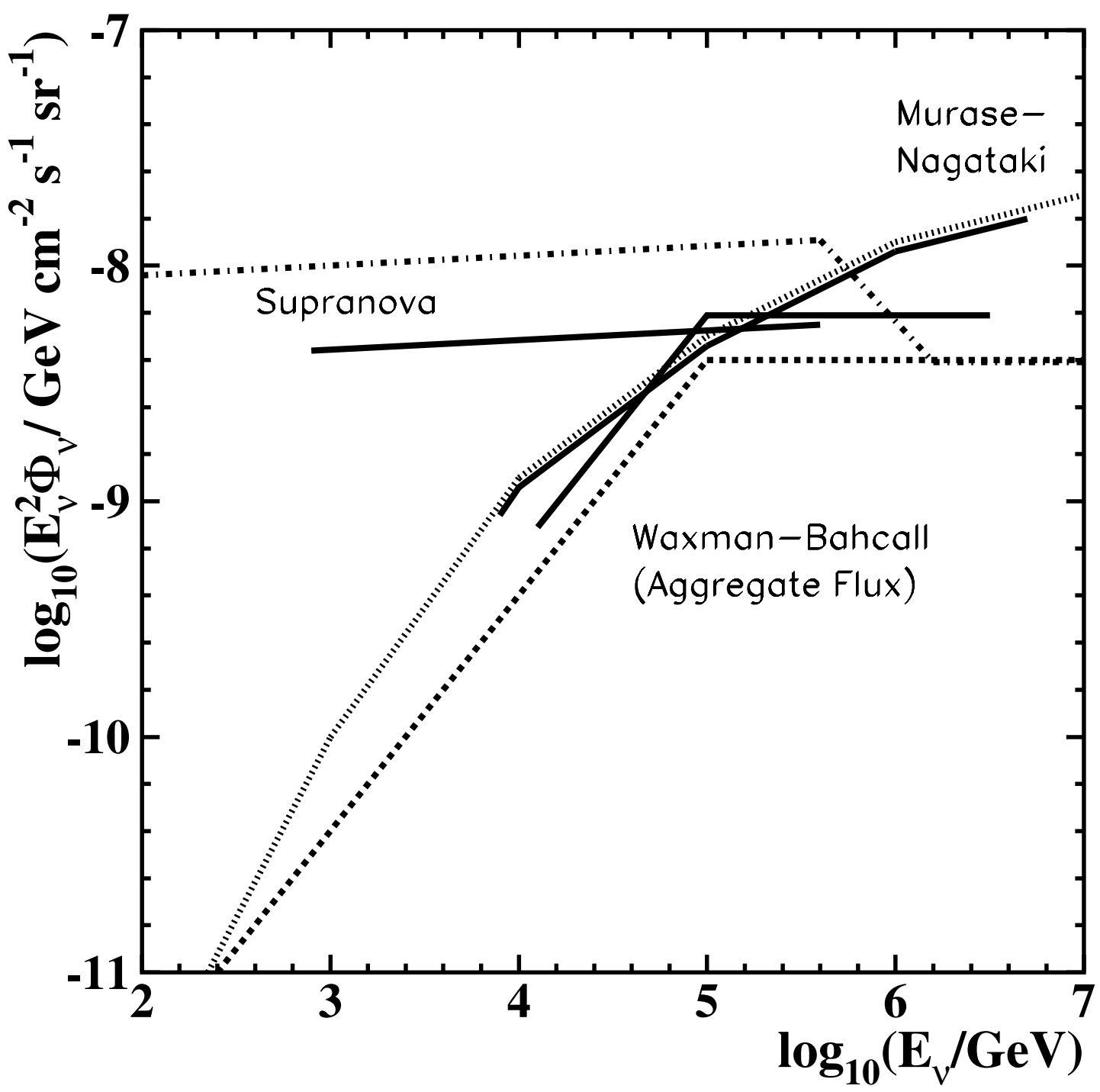

Fig. 9.- AMANDA flux upper limits (solid lines) for muon neutrino energy spectra predicted by the Waxman-Bahcall spectrum (Waxman 2003) (thick dashed line), the Razzaque et al. spectrum (Razzaque et al. 2003a) (dot-dashed line) and the Murase-Nagataki spectrum (Murase \& Nagataki 2006a) (thin dotted line). The central 90\% of the expected flux for each model is shown. For the Waxman-Bahcall model we include both long- and shortduration bursts; for the other spectra, only long-duration bursts are included. Including short-duration bursts would improve the flux upper limits by approximately $13 \%$. While our analysis was restricted to bursts located in the Northern Hemisphere $(2 \pi \mathrm{sr})$, all flux upper limits are for the entire sky $(4 \pi \mathrm{sr})$. 
This is an unedited preprint of an article accepted for publication in The Astrophysical Journal. The final published article may differ from this preprint. Copyright 2007 by The American Astronomical Society. Please cite as 'ApJ preprint doi:10.1086/'524920"'.

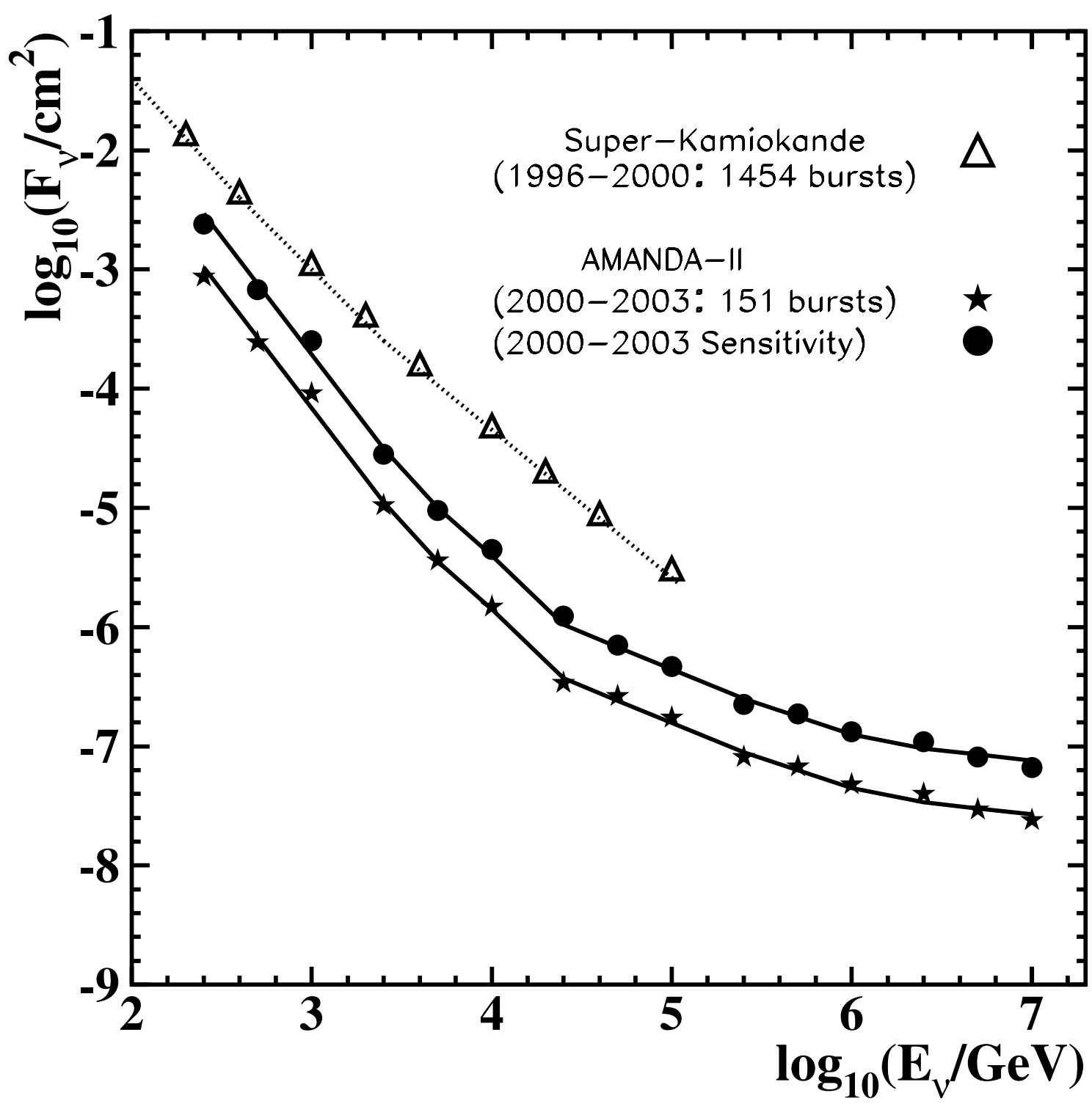

Fig. 10.- Green's Function Fluence Upper Limit for AMANDA's GRB analysis from 2000 to 2003. This fluence upper limit can be folded into any desired spectrum to provide a flux upper limit for that particular spectrum. 